\title{
Beyond water homeostasis: Diverse functional roles of mammalian aquaporins
}

\section{Philip Kitchen ${ }^{a}$, Rebecca E. Day ${ }^{\mathrm{b}}$, Mootaz M. Salman ${ }^{\mathrm{b}}$, Matthew T. Conner ${ }^{b}$, Roslyn M. Bill ${ }^{\mathrm{c}}$ and Alex C. Conner ${ }^{d^{*}}$}

${ }^{a}$ Molecular Organisation and Assembly in Cells Doctoral Training Centre, University of Warwick, Coventry CV4 7AL, UK

${ }^{b}$ Biomedical Research Centre, Sheffield Hallam University, Howard Street, Sheffield S1 1WB, UK

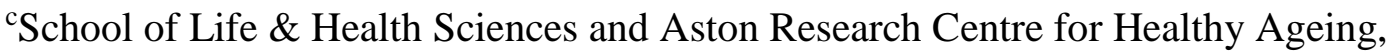
Aston University, Aston Triangle, Birmingham, B4 7ET, UK

${ }^{\mathrm{d}}$ Institute of Clinical Sciences, University of Birmingham, Edgbaston, Birmingham B15 2TT, UK

* To whom correspondence should be addressed: Alex C. Conner, School of Clinical and Experimental Medicine, University of Birmingham, Edgbaston, Birmingham B15 2TT, UK. 00441214158809 (a.c.conner@bham.ac.uk)

Keywords: aquaporin, solute transport, ion transport, membrane trafficking, cell volume regulation

The abbreviations used are: GLP, glyceroporin; MD, molecular dynamics; SC, stratum corneum; ANP, atrial natriuretic peptide; NSCC, non-selective cation channel; RVD/RVI, regulatory volume decrease/increase; TM, transmembrane; ROS, reactive oxygen species 


\section{Abstract}

BACKGROUND: Aquaporin (AQP) water channels are best known as passive transporters of water that are vital for water homeostasis.

SCOPE OF REVIEW: AQP knockout studies in whole animals and cultured cells, along with naturally occurring human mutations suggest that the transport of neutral solutes through AQPs has important physiological roles. Emerging biophysical evidence suggests that AQPs may also facilitate gas $\left(\mathrm{CO}_{2}\right)$ and cation transport. AQPs may be involved in cell signalling for volume regulation and controlling the subcellular localization of other proteins by forming macromolecular complexes. This review examines the evidence for these diverse functions of AQPs as well their physiological relevance.

MAJOR CONCLUSIONS: As well as being crucial for water homeostasis, AQPs are involved in physiologically important transport of molecules other than water, regulation of surface expression of other membrane proteins, cell adhesion, and signalling in cell volume regulation.

GENERAL SIGNIFICANCE: Elucidating the full range of functional roles of AQPs beyond the passive conduction of water will improve our understanding of mammalian physiology in health and disease. The functional variety of AQPs makes them an exciting drug target and could provide routes to a range of novel therapies. 


\section{Introduction}

The aquaporin (AQP) protein family is comprised of many small integral membrane proteins found in all phylogenetic kingdoms. There are up to 13 mammalian AQPs, which are found in most tissues with functions ranging from the regulation of renal water balance [1], brain-fluid homeostasis [2], triglyceride cycling between adipocytes and the liver [3] and structural integrity of the eye lens [4]. Because of this, understanding AQP function is crucial for the study of healthy ageing as well as the onset of many disease states such as brain swelling following stroke or head injury [5], nephrogenic diabetes insipidus [6, 7], cataracts [8], obesity [9], cancer cell proliferation and migration [10] and tumour angiogenesis [11]. Many of these functions and diseases involve either permeability of molecules other than water, or a function of the AQP other than facilitating membrane permeability.

Thirteen human AQPs have been discovered to date; they range in size (before posttranslational modification) from $27 \mathrm{kDa}$ (AQP8) to $37 \mathrm{kDa}$ (AQP7) and, for those proteins for which single channel permeability has been quantified, have a 100 -fold range in water permeability. A sub-set of these AQPs also function as channels for glycerol (and other solutes) and are referred to as (aqua)glyceroporins (GLPs). In humans, these are AQP3, -7, -9 and -10. AQP6 has also (controversially, see section 4) been shown to be permeable to glycerol, although phylogenetically it is a member of the water-selective AQP subfamily [12]. It also is notable for its unusual permeability properties (activation by low $\mathrm{pH}$ and anion permeability) and intracellular localization. The permeability properties of the mammalian AQPs are summarized in Table 1.

This review discusses how AQPs select and regulate the passage of solutes such as glycerol, urea and ammonia across cell membranes and the physiological relevance of this solute flow as well as putative functions of AQPs beyond facilitation of membrane permeability. 


\begin{tabular}{|c|c|c|c|c|}
\hline AQP & $\begin{array}{c}\text { Single channel water } \\
\text { permeability }\left(\times 10^{-14}\right. \\
\left.\mathrm{cm}^{3} \mathrm{~s}^{-1}\right)\end{array}$ & $\begin{array}{c}\text { Glycerol } \\
\text { permeability }\end{array}$ & $\begin{array}{c}\text { Urea } \\
\text { permeability }\end{array}$ & $\begin{array}{c}\text { Ammonia } \\
\text { permeability }\end{array}$ \\
\hline AQP0 & $\begin{array}{c}0.25[13] ; \\
\text { gated by calmodulin } \\
{[14] ; \mathrm{X}, \mathrm{PL}}\end{array}$ & $\begin{array}{c}\text { No [13]; } \\
\quad X\end{array}$ & $\begin{array}{c}\text { No }[15] ; \\
\quad X\end{array}$ & $\begin{array}{c}\text { No }[16] ; \\
X\end{array}$ \\
\hline AQP1 & $\begin{array}{c}6.0[13] \\
X\end{array}$ & $\begin{array}{c}\text { No [13]; } \\
\quad \mathrm{X}\end{array}$ & $\begin{array}{c}\text { No [17]; } \\
\mathrm{X}\end{array}$ & $\begin{array}{c}\text { No [17]; } \\
\quad \mathrm{X}\end{array}$ \\
\hline AQP2 & $\begin{array}{c}3.3[13] ; \\
X\end{array}$ & $\begin{array}{c}\text { No [13]; } \\
\mathrm{X}\end{array}$ & $\begin{array}{c}\text { No }[15] ; \\
X\end{array}$ & $\begin{array}{c}\text { No [16]; } \\
X\end{array}$ \\
\hline AQP3 & $\begin{array}{c}2.1[13] \\
\mathrm{X}\end{array}$ & $\begin{array}{c}\text { Yes [13]; } \\
\quad \mathrm{X}\end{array}$ & $\begin{array}{l}\text { Controversial } \\
{[15,17-19] ;} \\
\text { X }\end{array}$ & $\begin{array}{c}\text { Yes }[16,17] \\
\mathrm{X}\end{array}$ \\
\hline AQP4 & $\begin{array}{c}24[13] \\
X\end{array}$ & $\begin{array}{c}\text { No }[13] ; \\
X\end{array}$ & $\begin{array}{c}\text { No }[20] ; \\
X\end{array}$ & $\begin{array}{c}\text { No }[16] ; \\
X\end{array}$ \\
\hline AQP5 & $\begin{array}{c}5.0[13] \\
X\end{array}$ & $\begin{array}{c}\text { No [13]; } \\
\quad \mathrm{X}\end{array}$ & $\begin{array}{c}\text { No [21]; } \\
\quad X\end{array}$ & $\begin{array}{c}\text { No [16]; } \\
\quad X\end{array}$ \\
\hline AQP6 & $\begin{array}{l}\text { Low } \mathrm{pH} \text { and } \mathrm{Hg}^{2+} \text { induce } \\
\text { water permeability [22, } \\
\text { 23]. Basal permeability } \\
\text { either is very low [23, } \\
\text { 24] or zero }[25,26] \text {; } \\
\text { X }\end{array}$ & $\begin{array}{c}\text { Yes** [26]; } \\
\mathrm{X}\end{array}$ & $\begin{array}{c}\text { Yes** [26]; } \\
\mathrm{X}\end{array}$ & $\begin{array}{l}\text { Yes [16]; } \\
\quad \mathrm{X}\end{array}$ \\
\hline AQP7 & $\begin{array}{c}\text { Permeable [27]; no } \\
\text { quantitative data; } \\
\text { X }\end{array}$ & $\begin{array}{c}\text { Yes [27]; } \\
\quad \mathrm{X}\end{array}$ & $\begin{array}{c}\text { Yes [27]; } \\
\mathrm{X}\end{array}$ & $\begin{array}{c}\text { Yes [16]; } \\
\mathrm{X}\end{array}$ \\
\hline AQP8 & $\begin{array}{c}\text { Permeable }[28,29] ; \text { no } \\
\text { quantitative data; } \\
\text { X, PL }\end{array}$ & $\begin{array}{c}\text { No }[28,29] \\
\quad X, P L\end{array}$ & $\begin{array}{c}\text { Controversial } \\
\text { [28-30]; } \\
\text { X, PL } \\
\end{array}$ & $\begin{array}{c}\text { Yes }[17,31] \\
\text { X, Y }\end{array}$ \\
\hline AQP9 & $\begin{array}{c}\text { Permeable [32]; no } \\
\text { quantitative data; } \\
\text { PL }\end{array}$ & $\begin{array}{c}\text { Yes }[17,32] ; \\
\text { X, PL }\end{array}$ & $\begin{array}{c}\text { Yes }[17,32] ; \\
\text { X, PL }\end{array}$ & $\begin{array}{c}\text { Yes [17]; } \\
\quad \mathrm{X}\end{array}$ \\
\hline AQP10 & $\begin{array}{c}\text { Permeable [33]; no } \\
\text { quantitative data; } \\
\text { X }\end{array}$ & $\begin{array}{c}\text { Yes [33]; } \\
\text { X }\end{array}$ & $\begin{array}{c}\text { Yes [33]; } \\
\quad \mathrm{X}\end{array}$ & Unknown \\
\hline AQP11 & $\begin{array}{c}\text { Permeable [34], 2 [35]*; } \\
\text { M, V }\end{array}$ & $\begin{array}{c}\text { Yes** [36]; } \\
\text { M }\end{array}$ & Unknown & Unknown \\
\hline AQP12 & Unknown & Unknown & Unknown & Unknown \\
\hline
\end{tabular}

Table 1 - Water, glycerol, urea and ammonia $\left(\mathrm{NH}_{3}\right)$ permeabilities of known human AQPs. Quantitative water permeability is per AQP channel i.e. after subtraction of the basal permeability of the expression membrane and normalized to the number of AQP molecules. Expression systems: $X$-Xenopus oocytes, $P L$ - proteoliposomes, $M$ - mammalian cells, $V$-vesicles derived from membrane fragmentation of AQPexpressing mammalian cells, $Y$ - ammonia transport deficient yeast. . *estimated from membrane fragment vesicle water permeability relative to AQP1-containing vesicles and relative expression level. **These data have not been independently replicated. 


\section{The structural biology of the AQP family is established}

There is a large amount of medium-to-high-resolution structural data available for the AQP family (43 structures deposited in the Protein Data Bank for 11 different AQPs). These structures suggest that the AQPs share many common structural features. The AQP monomer is composed of six tilted transmembrane (TM) helices that surround a central cavity containing two helix-forming loops that enter and exit from the same side of the membrane (see Figure 1A). This central cavity is the pathway for water and solute transport. The two helical loops contain highly conserved asparagineproline-alanine (NPA) motifs. Crystallography and molecular dynamics (MD) simulations suggest that the asparagine residues act, in concert with surrounding backbone carbonyl groups, to enable water transport by providing hydrogen bonds to water molecules [37], thereby lowering the free energy penalty (caused by breaking of water-water hydrogen bonds) of removing a water molecule from bulk solution. Recent biophysical experiments comparing AQPs with gramicidin and the potassium channel KcsA [38] suggest that the number of pore-lining hydrogen-bonding sites for water determines the channel water permeability. In addition to providing hydrogenbonding sites, the asparagine residues help to orient the water molecules such that the hydrogen atoms point 'outwards' from the pore, creating a barrier to proton transport [39]. A recent sub-angstrom crystal structure of the Pichia pastoris AQY1 found overlapping water densities in the pore that, supported by MD simulations, suggested a correlated pairwise movement of water molecules through the extracellular end of the pore. This correlated motion may help to minimise water-water hydrogen bonds in this region, providing a further barrier to proton permeation, without compromising water transport [37].

Crystallographic analysis of the E. coli, GlpF (a GLP), suggests that the same asparagine residues also form hydrogen bonds with glycerol [40]. This is likely to be also true of other polar solutes that permeate GLPs.

AQPs have intracellular amino (N-) and carboxyl (C-) termini of varying lengths; AQP8 has a short C-terminus of 10-15 residues, whereas AQP4 has a large Cterminus consisting of $\sim 70$ residues. AQP11 has a large $\mathrm{N}$-terminal tail of $\sim 40$ residues, whereas AQPs 1,2 and 5 have short $\mathrm{N}$-termini of $<10$ residues. In contrast to the structural conservation of the TM region of AQPs, much less is known about the structure of the termini. This is because the majority of AQP crystal structures are obtained using constructs in which the $\mathrm{N}$ - and $\mathrm{C}$-terminal tails of the protein have been truncated due the difficulty of obtaining well-diffracting crystals using fulllength AQP molecules [41]. This suggests an inherent structural flexibility in these regions of $\mathrm{AQP}$ molecules and indeed a recent X-ray structure of AQP2, which included $\sim 20$ residues of the (truncated) C-terminal tail, found this fragment of the Cterminus in four strikingly different conformations in each of the four AQP2 monomers within the tetrameric unit cell [42].

AQPs are generally agreed to form tetramers [43-45] assembled around a central axis perpendicular to the plane of the membrane (see Figure 1B). A fifth, hydrophobic pore appears to form around this central axis with each of the monomers contributing one of the four 'walls' of the pore. The function, if any, of this fifth pore is not yet known, although postulated functions will be discussed in later sections. Some biochemical studies have suggested that tetramers formed by GLPs have reduced stability compared to tetramers formed by AQPs with strict water selectivity $[46,47]$. 


\section{The principles of solute selectivity by AQPs remain to be established}

All AQPs (except AQP12, which has yet to be characterized) transport water (Table 1), while some also transport glycerol. The glycerol permeable GLPs (e.g. human AQPs 7, 9 and 10) are also permeable to urea. The urea permeability of AQP3 is controversial, with some studies reporting urea transport and others no transport [15, 17-19]. This may be due to methodological differences, and is discussed in detail in section 4.2.1.

Of the mammalian GLPs AQP3, -7 , and -9 are also permeable to ammonia. AQP8 is the only glycerol impermeable mammalian AQP that is permeable to ammonia (excluding 11 and 12, whose glycerol and/or ammonia permeabilities are currently unknown). The ammonia permeability of the most recently discovered member of the GLP group, AQP10, is still unknown.

AQP11 and -12 are the most recently discovered members of the AQP family [48, 49]; due to this and their localisation to intracellular membranes, non-water permeability experiments and functional studies have yet to be reported for AQP11 and AQP12 respectively. There is one report that AQP11 increased the glycerol permeability of an adipocyte cell line [36], but this permeability of AQP11 has yet to be replicated.

The 'size exclusion' model of AQP selectivity posits a correlation between the size of channel entrance and the permeability of the channel to different polar molecules. Notably, this model is based on in silico and crystallographic experiments, but minimal in vitro experiments; it is therefore unclear whether this model is broadly applicable to the AQP family as a whole. A conserved arginine residue (in the second helical loop directly after the NPA motif) forms part of a channel constriction known as the aromatic/arginine ( $\mathrm{ar} / \mathrm{R})$ selectivity filter (see Figure 1A). In water-selective AQPs, the other components of this filter are a phenylalanine in the top half of the pore-facing side of transmembrane helix 2 (TM2) and a histidine in a similar position in TM5. In glycerol and urea permeable AQPs, this histidine is replaced by a small amino acid residue such as glycine (AQP3, -7, -10) or alanine (AQP9), although if the histidine is mutated to alanine in AQP1 it does not become a glycerol channel. If both aromatic members of the filter ( $\mathrm{H}$ and $\mathrm{F}$ ) are mutated to alanine, AQP1 functions as a urea channel and to a lesser extent as a glycerol channel [50]. Based on this observation and molecular simulations of AQP1 and the E. coli GLP, GlpF [50, 51], it has been suggested that the cross-sectional area of the pore at the ar/R region determines AQP selectivity for neutral polar solutes. Additionally, based on crystallographic analysis of GlpF and the bacterial water-selective AQP, AQPZ, it has been suggested that the positioning of the ar/R residues by the surrounding unstructured loops has a role in determining the channel size [52]. In AQP8, the only glycerol-impermeable, ammonia-permeable mammalian AQP, the third member of the filter is likely an isoleucine residue (based on sequence alignment), which is slightly larger than alanine or glycine. Overall, current thinking on solute selectivity suggests that the aromatic residues of the ar/R filter are important for solute exclusion in the water selective AQPs, but further molecular factors may be involved in mediating solute selectivity. 


\section{Physiological solute transport by AQPs \\ 4.1 Glycerol transport}

The movement of glycerol around the body is predominantly thought to include glycerol release from fat tissue through $\mathrm{AQP7}$, entry into the liver through $\mathrm{AQP9}$ and movement in the skin and kidneys through AQP3.

\subsubsection{AQP7}

AQP7 is expressed in adipose tissue in adipocytes [53] and capillary endothelia [54]. Upon hypoglycaemia, triglycerides are broken down within adipocytes to glycerol and free fatty acids [55]. Adrenaline, the plasma level of which is elevated upon hypoglycaemia, caused translocation of AQP7 from intracellular membranes to the plasma membrane [53]. Adrenaline signals through the $\beta 3$-adrenergic receptor to initiate adipocytic lipolysis [56] making this the most likely link between adrenaline and AQP7 translocation although this has yet to be verified. Recent work has also suggested that $\mathrm{AQP7}$ can be relocalized to lipid droplets by noradrenaline, possibly in a PKA dependent manner [57], raising the possibility of differential effects of lipolysis-inducing hormones on AQP7 localization.

The glycerol liberated from triglyceride storage is released from adipocytes [58] and plasma glycerol becomes elevated. In AQP7 -/- mice, adipocyte plasma membrane glycerol permeability was reduced threefold [59]. Plasma glycerol levels in the fasting state and in response to $\beta 3$-adrenergic agonist were reduced and adrenaline-induced glycerol secretion by cultured mouse adipocytes (differentiated 3T3-L1 cells) was reduced approximately two-fold [60]. These results suggest that the major pathway for glycerol efflux from adipocytes after lipolysis is AQP7.

AQP7 -/- mice develop adipocyte hypertrophy and subsequent obesity in adulthood[59]. A loss of function mutation in the human AQP7 gene has been discovered $(\mathrm{G} 264 \rightarrow \mathrm{V})$ although this genotype was not correlated with obesity in humans [61]. This may be due to the presence of AQP10 in human adipocyte membranes, which was found to contribute $\sim 50 \%$ of the water and glycerol permeability of healthy human adipocyte plasma membrane vesicles [62]. Mouse AQP10 is a pseudogene [63] and therefore this secondary route for glycerol efflux does not exist in mice.

The serum concentration of glycerol in humans under normal physiological conditions is typically between 0.05 and $0.1 \mathrm{mM}$ [64] and increases several-fold upon fasting (due to glycerol and free fatty acid liberation from adipose triglyceride storage) [65]. Glycerol is almost completely reabsorbed by the kidneys [66] (unless it is raised above $\sim 0.3 \mathrm{mM}[67]$ ), suggesting the existence of a glycerol reabsorption pathway. AQP7 is expressed in the proximal tubule of the kidney. AQP7 -/- mice showed marked glyceroluria in comparison to wild type ( $\sim 400$-fold increase in urine glycerol) [68]. Human children homozygous for the AQP7 G264V mutant were found to have hyperglyceroluria, with a $~ 1000$-fold increase in urine glycerol when compared to heterozygous familial controls [69]. The G264V mutant has been shown to have no activity as a glycerol or water channel in Xenopus oocytes [61], although plasma membrane expression was not verified in this study so that it could not differentiate between a non-functional channel and an incorrectly localised channel. The mutation disrupts a conserved GxxxG motif. These are important motifs for 
transmembrane helix-helix interactions because they allow close contact between the backbone atoms of the two helices of an interacting pair [70]. It may be that the protein is incorrectly trafficked and localised in intracellular membranes due to an inability to form tetramers or an interaction required for membrane trafficking. Regardless of the loss of function mechanism, these data strongly suggest that AQP7 is the TM glycerol reabsorption pathway in the renal proximal tubule.

\subsubsection{AQP9}

AQP9 is expressed in the liver [71], primarily in the hepatocytes with the strongest expression at the sinusoidal surface [72] (the liver sinusoids are continuous with the hepatic artery and portal vein). Upon fasting, glycerol released into the plasma from adipocytes is taken up by hepatocytes and used as a substrate for gluconeogenesis [73].

In rats, AQP9 protein expression is increased up to 20-fold after 24-96 hours fasting [32]. In mice, AQP9 expression measured in purified hepatocyte plasma membrane vesicles increased 10-fold after 18 hours fasting. This 10-fold increase was accompanied by a two-fold increase in hepatocyte plasma membrane glycerol permeability, which was reversed by addition of phloretin (an apple polyphenol that inhibits AQP9, AQP3 and several non-AQP membrane channel proteins including the SGLT1\&2 glucose transporters and UT-A urea channels [74]). The increase was abolished in AQP9 -/- mice [75]. Fasted AQP9 -/- mice have elevated plasma glycerol compared to wild-type $[75,76]$. In addition, plasma glucose concentration was decreased [76], indicating a gluconeogenetic deficiency. This data supports a physiological role for AQP9-mediated glycerol uptake by hepatocytes during fastinginduced hypoglycaemia.

Although the AQP9-mediated diffusion of glycerol into hepatocytes forms a sizeable proportion of the glycerol uptake in the fasting state ( $\sim 50 \%$ in fasting mice), the fact that the membrane permeability to glycerol only doubles upon such a large increase in AQP9 expression suggests that there is also an AQP9-independent route for glycerol uptake. Whether this is directly across the lipid bilayer or through a phloretin insensitive glycerol channel is not clear.

$\mathrm{CD}^{+}$(cytotoxic) $\mathrm{T}$ cells are white blood cells that facilitate the destruction of infected or otherwise damaged cells. During infection, antigen-specific $\mathrm{CD}^{+}$cells proliferate and differentiate into effector cells, which are involved in combatting infection. After pathogen clearance, most of the effector cells die and a small population remains as memory T cells, which can survive for decades [77]. In mouse memory cells, AQP9 expression was upregulated post-infection via IL-7 signalling, allowing cells to import glycerol for triglyceride synthesis. AQP9 -/- T cells had reduced long-term survival compared to $+/+$ cells and the long-term survival of the $+/+$ cells could be inhibited by phloretin [78]. This suggests that AQP9 expression can act as a metabolic switch, enabling long-term survival of memory $\mathrm{T}$ cells by enabling triglyceride synthesis to build up an energetic reserve, allowing cell survival in nutrient poor conditions.

Similarly to that seen in hepatocytes, intracellular glycerol in $\mathrm{CD}^{+} \mathrm{T}$ cells was reduced by $\sim 50 \%$ in the AQP9 -/- cells, again suggesting a secondary route for glycerol uptake. 


\subsubsection{AQP3}

AQP3 is expressed in the skin in keratinocytes below the stratum corneum (SC). It is most strongly expressed at the plasma membrane in these cells with some intracellular labelling reported in cells of the basal layer [79]. The ability of the epidermis to maintain hydration is impaired in AQP3 -/- mice. In dry conditions, AQP3 -/- mice showed comparable (reduced) levels of SC hydration to wild-type mice, whereas at normal humidity, SC hydration was lower in the AQP3 -/- mice [80]. Elevated humidity (which prevents water loss via evaporation) did not correct the deficiency, which suggests that the primary role of AQP3 in skin hydration is not to provide water to replace that lost by evaporation.

SC glycerol concentration in AQP3 -/- mice is reduced to $40 \%$ of that of wild-type, with no significant difference in the levels of other osmolytes (ions, glucose, urea, lactate and free amino acids) [81]. Glycerol acts as a humectant (a 'water-retaining' osmolyte), which may be the mechanism by which it maintains skin hydration. It has also been suggested that glycerol may prevent SC water loss by inhibiting the phase transition of intercellular lipids from the liquid crystalline to the solid phase [82] due to the fact that breaks in a solid crystal lattice increase the water permeability in in vitro models of the SC intercellular lipid barrier [83]. The rate of transport of glycerol from blood to the SC was reduced in AQP3 -/- mice resulting in reduced lipid biosynthesis[84]. This suggests a further role for glycerol in maintaining skin hydration by allowing the maintenance of the SC lipid barrier.

Further evidence for the role of AQP3-mediated glycerol transport is provided by the fact that topical or systemic administration of glycerol was found to correct the skin deficiencies in AQP3 -/- mice [84].

\subsection{Urea transport}

Urea is produced in the liver (as a non-toxic carrier of waste nitrogen) from ammonia, which is a neurotoxic product of protein degradation. Ammonia causes cell death of astrocytes by stimulating the mitochondrial permeability transition [85] (opening of promiscuous mitochondrial membrane channels that leads to apoptosis or necrosis, depending on cellular ATP availability [86]), although the mechanism by which this happens is not clear. An adult human excretes about $25 \mathrm{~g} /$ day of urea in the urine, and urea transport in the kidney is vital for the urinary concentrating mechanism [87]. The physiological roles of urea transport by AQPs are less clear than those of water and glycerol transport.

\subsubsection{Which mammalian AQPs are urea channels?}

AQPs 7 [27], 9 [88] and 10 [33] have been shown to be urea permeable and there is a consensus in the literature on the urea permeability of these AQPs. For example, AQP9 is a urea channel in the liver expressed in hepatocytes at the sinusoidal surface. Urea permeability measurements performed on mouse hepatocyte plasma membrane vesicles of AQP9 -/- and urea transporter type A1/3 (UT-A1/3) -/- mice showed that AQP9 contributes $\sim 30 \%$ to mouse hepatocyte membrane permeability and a member(s) of the UT-A family contributes $40 \%$ [91]. However, AQP9 -/- mice did not demonstrate any deficiency in urea clearance from hepatocytes in a state that promotes elevated hepatic urea production (high protein diet), suggesting that AQP9 and UT-A provide redundant pathways for urea transport from hepatocytes to the blood. 
However, there is conflicting evidence in the literature on whether AQPs 3 and 8 are urea channels. Early work on AQP3 suggested that rat AQP3 was urea permeable, with expression of AQP3 in Xenopus oocytes increasing urea uptake twofold after 30 minute incubations of oocytes with radiolabelled urea [89] or threefold in oocyte swelling assays [18]. Further studies on rat AQP3 found no urea transport using similar oocyte volumetric techniques $[15,17]$. These studies differed in that the former used $165 \mathrm{mM}$ urea whereas the latter two used $20 \mathrm{mM}$ urea. It may be that AQP3 urea transport is so slow that at $20 \mathrm{mM}$ it does not induce large enough volume changes to be measured on the timescale of an oocyte swelling experiment ( 1 min), or that the transport is non-linear, although this seems unlikely given the linear nature of water and glycerol transport by AQP3 [90].

In one study, human AQP3 was used a positive control for AQP urea permeability. 1 $\mathrm{mM}$ of urea was added to AQP3-expressing oocytes and after a 10 minute incubation, the oocytes had an intracellular urea concentration of $\sim 75 \mu \mathrm{M}$ (assuming an oocyte volume of $1 \mu \mathrm{L}$ ) [19]. This is $\sim 10 \%$ of the expected equilibrium value, and the fact that it is still so far from equilibrium even after 10 minutes suggests that urea transport through AQP3, whilst non-zero, is very slow. This may explain the different results between short (typically $\sim 1 \mathrm{~min}$ ) volumetric experiments and the longer timescale radiolabelled solute uptake experiments.

Early work on mouse AQP8 suggested that it was urea permeable [30], whereas rat AQP8 was not [29], both using radiolabelled solute uptake measurements in Xenopus oocytes. Work on purified rat, mouse and human APQ8 in proteoliposomes suggested that neither rat nor human AQP8 were urea permeable [28]. This study did not report mouse urea permeability due to liposome swelling discrepancies, probably caused by the ionic detergent required to solubilize mouse AQP8.

There is considerable interspecies amino acid sequence variability for AQP8 (e.g. $74 \%$ identity between human and mouse AQP8, c.f. 94\% for AQP1 and 93\% for AQP4). Interestingly, one difference between human and mouse AQP8 is a residue that is predicted to be pore-lining (based on a homology model to bovine AQP0 [28]) and situated at the ar/R filter, G207 (in human; A205 in mouse). The idea of speciesspecific differences in permeability of AQP8 is intriguing, but studies performed in parallel in the same experimental system are required to validate this.

\subsection{Ammonia transport}

Ammonia is produced as a by-product of protein breakdown and quickly converted to urea via the hepatic urea cycle to prevent ammonia neurotoxicity. It is important for control of acid-base balance in the kidney, where ammonia synthesis and excretion are tightly regulated and change in response to acid- or alkalosis [92].

Members of the GLP subfamily, AQPs 3 [17], 7 [16] and 9 [17], have been reported to be permeable to ammonia, as has AQP8 $[17,93]$. There is also evidence of ammonia permeability of AQPs 1, 6 and 7 using microelectrode measurements of oocyte surface $\mathrm{pH}[16]$. The physiological relevance of ammonia permeability of AQPs is unclear.

AQP8 -/- mice have only the very mild phenotypic abnormality of slight hypertriglyceridaemia after three weeks on a high (50\%) fat diet [94]. AQP8 is expressed in the inner mitochondrial membrane of hepatocytes [95] and increased the 
transport of an ammonia analogue into AQP8-expressing $S$. cerevisiae and rat hepatocyte mitochondria by three-fold. However, AQP8 -/- mice do not show any impairment in ammonia clearance under physiological conditions or when chronically or acutely loaded with ammonia [96]. This suggests a secondary (non-AQP) pathway for ammonia, which either provides the majority of ammonia permeability in these tissues, or is up-regulated in ammonia-permeable AQP knockouts. The proteins associated with the Rhesus (Rh) blood group system have been shown to function as ammonia channels [97]. Several of these are expressed in the liver [98] ( $\mathrm{RhB}$ and $\mathrm{RhC}$ ) and the kidneys (again $\mathrm{RhB}$ and $\mathrm{RhC}$ ), where ammonia has an important role in acid-base balance [99]. Rh -/- mice have been generated, however these studies have focused on the erythrocytic Rh proteins [100, 101]. Phenotype analysis of RhB and $\mathrm{RhC}$-/- organisms and double AQP/Rh knockouts could provide an answer. Knockdown of AQP8 in primary rat hepatocytes by $\sim 80 \%$ reduced ammonium chloride-induced ureagenesis by $30 \%$ and abolished glucagon-stimulated increases in ureagenesis [102]. AQP8 knockdown in a human proximal tubule cell line decreased the rate of ammonia excretion by $31 \%$ at $\mathrm{pH} 7.4$ and by $90 \%$ at $\mathrm{pH} 6.9$ [103], suggesting that AQP8 ammonia permeability might be required for renal ammonia excretion and be involved in the renal adaptive response to acidosis. Furthermore, acid-induced downregulation of AQP8 by $30 \%$ in primary rat hepatocytes was correlated with a $31 \%$ reduction in hepatocyte ureagenesis, and AQP8 downregulation was correlated with reduced liver urea content in rats subjected to seven days of acidosis [104]. These data support the idea of a physiological role for AQP8 in either plasma membrane diffusion of ammonia, mitochondrial ammonia transport, or both in support of renal and hepatic ammonia handling.

AQP3 -/- mice have several physiological abnormalities including reduced skin elasticity [80] and polyuria [105], but these can all be explained in terms of reduced glycerol and water permeability, so it is unclear whether AQP3 ammonia permeability has any physiological function.

AQP7 is present in adipocytes. It has been shown in humans that during intense exercise, adipose tissue removes ammonia from the plasma and increases its glutamine/glutamate ratio[106], suggesting incorporation of ammonia into glutamine through glutamine synthase as a secondary detoxification pathway in support of the hepatic urea cycle at times of elevated plasma ammonia. Whether AQP7 contributes to this ammonia uptake is yet to be investigated.

\subsection{Carbon dioxide transport}

The majority of carbon dioxide $\left(\mathrm{CO}_{2}\right)$ produced by cellular metabolism $(\sim 70 \%)$ is transported to the lungs for expulsion from the body via the bicarbonate $\left(\mathrm{HCO}_{3}{ }^{-}\right)$ system. Briefly, carbon dioxide diffuses out of the cells in which it is produced and into the plasma. It moves down its concentration gradient into erythrocytes (red blood cells), where carbonic anhydrase catalyses conversion into carbonic acid. Upon dissociation $\left(\mathrm{H}_{2} \mathrm{CO}_{3} \rightarrow \mathrm{H}^{+}+\mathrm{HCO}_{3}{ }^{-}\right)$, the $\mathrm{HCO}_{3}{ }^{-}$ion is exchanged across the erythrocyte membrane for a chloride ion and the proton binds to haemoglobin [87].

There has been speculation that $\mathrm{CO}_{2}$ transport across the plasma membrane of erythrocytes might be aided by channel proteins $[107,108]$. There is conflicting data on whether an AQP contributes to this potential $\mathrm{CO}_{2}$ pathway. AQP1 is expressed at the plasma membrane of erythrocytes [109]. Human AQP1 has been reported to 
increase the $\mathrm{CO}_{2}$ permeability of Xenopus oocytes four-fold in the presence of carbonic anhydrase suggesting that AQP1 functions as a $\mathrm{CO}_{2}$ channel [110]. Further work ruled out the possibility of AQP1 increasing the oocyte membrane permeability by altering local lipid composition or structure, interaction between AQP1 and carbonic anhydrase or up-regulating a native $\mathrm{CO}_{2}$ channel by use of a mercurial AQP1 inhibitor (pCMBS) and the AQP1 C189S mutant, which is insensitive to mercury [111].

AQP1 -/- human erythrocytes (Colton-null cells) retain $\mathrm{HCO}_{3}{ }^{-}$permeability, but have a $60 \%$ reduction in $\mathrm{CO}_{2}$ permeability when compared to human erythrocytes expressing AQP1 (i.e. not Colton-null) [112]. However, the $\mathrm{CO}_{2}$ permeability of AQP1 -/- mouse erythrocytes from AQP1 knockout mice was not different to wildtype [113].

Stopped flow experiments with AQP1 reconstituted into liposomes have also given conflicting results. AQP1 from human blood increased the $\mathrm{CO}_{2}$ permeability of liposomes four-fold[114], whereas mouse AQP1 showed no increase[113]. This agrees qualitatively with the results from measurements on intact erythrocytes in that human AQP1 appears to increase $\mathrm{CO}_{2}$ permeability of the membrane, whereas mouse AQP1 does not. This may be due to methodological differences, but it also raises the intriguing possibility that human $\mathrm{AQP} 1$ is $\mathrm{CO}_{2}$ permeable whereas mouse AQP1 is not.

It is possible that if $\mathrm{CO}_{2}$ permeates $\mathrm{AQP} 1$, it does so through the central tetrameric pore rather than the monomeric water pore. Molecular dynamics simulations using a variety of enhanced sampling methods and unbiased simulation consistently suggest that the free energy barrier to permeation of the central, hydrophobic pore of AQP1 is considerably smaller than the barrier to permeation of the water pores [115-117], probably due to the water-protein hydrogen bonds at the selectivity filter and NPA sites that need to be disrupted in order for a $\mathrm{CO}_{2}$ molecule to traverse the water pore. Simulation of model bilayers suggest that the free energy barrier for passage of $\mathrm{CO}_{2}$ directly across the membrane is much smaller than for permeation through AQP1, and in this kind of system, AQP expression would probably serve to decrease the $\mathrm{CO}_{2}$ permeability by reducing the surface area available for diffusion directly across the membrane $[115,116]$. However, it is not clear how good of a model a single species phospholipid bilayer is for a cellular membrane. It is possible that a simulated bilayer containing a mixture of lipid species along with sterols would display different resistance to $\mathrm{CO}_{2}$ passage, and biophysical measurements of $\mathrm{CO}_{2}$ diffusion into artificial lipid vesicles showed that addition of cholesterol could reduce membrane $\mathrm{CO}_{2}$ permeability by up to 100 -fold [118]. This agrees qualitatively with $\mathrm{MD}$ simulations that include cholesterol [119]. In addition to $\mathrm{CO}_{2}, \mathrm{MD}$ simulations have also suggested that the central pore could be permeable to molecular oxygen [115] and there is some evidence that AQP1 overexpression could accelerate cellular hypoxia [120], although whether this was a direct or indirect effect was not clear.

It seems apparent that $\mathrm{AQP} 1$ can conduct $\mathrm{CO}_{2}$ (possibly along with $\mathrm{AQP} 0,5,6$ and 9 and the M23 isoform of AQP4 [16]), however the physiological relevance (if any) of $\mathrm{AQP} \mathrm{CO} 2$ permeability is yet to be demonstrated. There is also a similar debate in the plant AQP field on whether AQPs might contribute to regulating CO2 levels for photosynthesis [121]. A recent review dedicated to AQPs and $\mathrm{CO}_{2}$ permeability of 
biological membranes [122] concluded that "the debate about the mechanism of membrane $\mathrm{CO} 2$ diffusion continues and it is difficult to draw general conclusions". This is still very much an open question.

\subsection{Hydrogen peroxide transport}

Superoxide $\left(\mathrm{O}_{2}^{-}\right)$molecules are produced in the mitochondria as a by-product of ATP synthesis (particularly by complexes I and III in the electron transport chain) and by the NADPH oxidase (NOX) family of enzyme complexes, which couple intracellular oxidation of NADPH to extracellular production of superoxide $\left(\mathrm{O}_{2}^{-}\right)$molecules. High levels of reactive oxygen species (ROS) derived from these pathways are well known to cause cellular damage and even death and mitochondrial ROS were long considered to be purely harmful by-products of an imperfect metabolic system. However, work in the last decade has shown that they are crucial for a variety of physiological processes including adaptation to hypoxia, immune function and regulation of autophagy (probably mediated by redox modification of cysteine residues) [123], so clearly there is a need for tight regulation of ROS levels. Superoxide undergoes disproportionation to molecular oxygen and hydrogen peroxide $\left(\mathrm{H}_{2} \mathrm{O}_{2}\right)$, which can be catalysed by the enzyme superoxide dismutase [124], and the highly reactive $\mathrm{H}_{2} \mathrm{O}_{2}$ can then go on to form a variety of further ROS.

$\mathrm{H}_{2} \mathrm{O}_{2}$ and $\mathrm{H}_{2} \mathrm{O}$ have similar molecular sizes, dipole moments and hydrogen bonding capacities [125], therefore it would not be surprising to find that some AQPs could transport $\mathrm{H}_{2} \mathrm{O}_{2}$. The half-life for $\mathrm{H}_{2} \mathrm{O}_{2}$ in cells is very short (e.g. $\sim 1 \mathrm{~ms}$ in lymphocytes [126]), making biophysical experiments of the kind used for e.g. glycerol or urea permeation very difficult. Growth assays using $\mathrm{H}_{2} \mathrm{O}_{2}$ transportdeficient yeast are typically used, and reduced growth after expression of an AQP is interpreted as an AQP-mediated increase in membrane $\mathrm{H}_{2} \mathrm{O}_{2}$ permeability causing cellular damage and/or metabolic disruption. Using this technique, human AQP8, rat (but not human) AQP1 and several mutants thereof were shown to be $\mathrm{H}_{2} \mathrm{O}_{2}$ permeable [127]. Based on this data, the authors suggested that all AQPs function as $\mathrm{H}_{2} \mathrm{O}_{2}$ channels. It is not clear whether this is a valid generalization, but clearly some mammalian AQPs are $\mathrm{H}_{2} \mathrm{O}_{2}$ channels.

Imaging studies using a novel $\mathrm{H}_{2} \mathrm{O}_{2}$ sensitive fluorescent dye in transiently transfected HEK293 cells demonstrated $\mathrm{H}_{2} \mathrm{O}_{2}$ permeability for human AQP8 and AQP3 (but not AQP1). Furthermore, AQP3 overexpression in HeLa cells allowed the cells to respond to serum starvation via ROS mediated activation of the AKT (protein kinase B) signalling pathway, whereas cells not expressing AQP3 could not. Knockdown of AQP3 in a colon cancer cell line inhibited the AKT response to epidermal growth factor (EGF) [128], which can initiate ROS signalling via activation of NOX complexes. This suggests that AQP3 is required for uptake of NOX-generated $\mathrm{H}_{2} \mathrm{O}_{2}$ in EGF signalling.

$\mathrm{T}$ cell migration along chemokine gradients is vital for correct immune responses. $\mathrm{T}$ cells from AQP3 -/- mice had 40-60\% reductions in migration distance in response to a variety of chemokines and had a $\sim 50 \%$ reduction in chemokine-induced transendothelial migration. Activation of Cdc42, a small GTPase involved in cytoskeletal reorganization associated with chemotaxis, was completely inhibited in the AQP3 -/- cells. Treatment with extracellular catalase, which catalyses $\mathrm{H}_{2} \mathrm{O}_{2}$ breakdown to $\mathrm{H}_{2} \mathrm{O}$ and $\mathrm{O}_{2}$, prevented the $\mathrm{Cdc} 42$ response in AQP3 +/+ cells and 
treatment with high concentration $(100 \mu \mathrm{M})$ of exogenous $\mathrm{H}_{2} \mathrm{O}_{2}$ recovered both the $\mathrm{Cdc} 42$ response and the T cell migration in AQP3 -/- cells [129]. Together, these data suggest that AQP3 is required for uptake of extracellular $\mathrm{H}_{2} \mathrm{O}_{2}$ (probably NOXgenerated) in the $\mathrm{T}$ cell chemotactic response.

Intracellular ROS levels can be elevated in leukaemia cells and NOX-derived ROS can activate leukaemia cell survival pathways. In a leukaemia cell line, shRNAmediated knockdown of AQP8 reduced and overexpression increased intracellular $\mathrm{H}_{2} \mathrm{O}_{2}$ content as well as $\mathrm{H}_{2} \mathrm{O}_{2}$ uptake after 10 mins in $100 \mu \mathrm{M} \mathrm{H}_{2} \mathrm{O}_{2}$. Furthermore, cellular glucose uptake and proliferation were correlated with intracellular $\mathrm{H}_{2} \mathrm{O}_{2}$ and AQP8 expression [130], suggesting that AQP8 can facilitate increased metabolism and proliferation in leukaemia.

AQP8 is also expressed in inner mitochondrial membranes (IMM) in hepatocytes. Knockdown of AQP8 by $60 \%$ using siRNA in a hepatocyte cell line caused a twofold increase in mitochondrial ROS concentration and a $45 \%$ decrease in $\mathrm{H}_{2} \mathrm{O}_{2}$ output in isolated mitochondria. This was correlated with an $80 \%$ depolarization of the mitochondrial membrane, which was reversible by cyclosporin A (an inhibitor of the mitochondrial permeability transition) or a mitochondrial antioxidant, and a $\sim 30 \%$ reduction in cell viability [131]. This suggests that AQP8-mediated $\mathrm{H}_{2} \mathrm{O}_{2}$ release from hepatic mitochondria acts as a mechanism to minimize mitochondrial oxidative stress.

AQP11 is localized to the ER in the renal proximal tubule. The C227S mutation of AQP11 caused proximal tubule injury and eventual renal failure in mice [132]. Mice heterozygous for the AQP11 C227S mutant were predisposed to glucose-induced accumulation of ROS in the proximal tubule and reduction of kidney function. Inhibition of glucose uptake by the non-specific inhibitor phlorizin or antioxidant (sulforaphane) treatment was able to protect kidney function. This effect was reproduced in a proximal tubule cell line, in which siRNA-mediated knockdown of AQP11 increased intracellular ROS concentration twofold in the absence of glucose and fourfold in the presence of glucose [133]. It is not clear whether AQP11 was controlling intracellular ROS accumulation by acting as an ER $\mathrm{H}_{2} \mathrm{O}_{2}$ channel, or whether this was an indirect effect.

Taken together, these examples provide clear evidence for AQP-mediated $\mathrm{H}_{2} \mathrm{O}_{2}$ membrane permeability and suggest physiological roles in redox signalling via uptake of NOX-derived $\mathrm{H}_{2} \mathrm{O}_{2}$ and in cellular mechanisms for minimizing oxidative stress.

A recent review of $\mathrm{H}_{2} \mathrm{O}_{2}$ permeability of AQPs suggested that rat AQP1 may be permeable to $\mathrm{H}_{2} \mathrm{O}_{2}$ whereas human AQP1 is not [134]. The authors of the cited study [127] suggest that the difference is due to differences in plasma membrane localization, although surface expression was not measured directly. This difference is particularly interesting given the speculation that we made regarding differences in $\mathrm{CO}_{2}$ permeability between human and mouse AQP1 and the possible differences in AQP8 urea permeability between species and raises the important point that despite high levels of conservation between AQPs from different mammals, they are not exactly the same proteins (e.g. rAQP1 and hAQP1 differ by 18 residues), and direct comparison between AQPs from different mammals in different experimental systems may not always be appropriate. 


\section{The physiological role of AQP6: An unusual AQP}

AQP6 is expressed primarily in kidney epithelia, where it is expressed only in intracellular membranes. Immunostaining was observed in podocyte intracellular vesicles, sub-apical vesicles in straight proximal tubule cells, and in both sub-apical and sub-basolateral domains within type A (acid-secreting) intercalated cells in the collecting duct[135].

AQP6 has low intrinsic water permeability: The membrane water permeability of Xenopus oocytes expressing AQP6 was increased less than threefold [22, 136], and in some experiments, not at all $[25,26]$. In contrast to other members of the AQP family, mercury increases rather than inhibits AQP6 water permeability, which is reversibly increased approximately tenfold upon $\mathrm{HgCl}_{2}$ application. Low $\mathrm{pH}$ activated a reversible anion permeability of AQP6 [22] which was also shown to be tenfold more permeable to nitrate than chloride [137]. In rat collecting duct, intercalated cells, AQP6 co-localises with $\mathrm{H}^{+}$-ATPase in intracellular vesicles [22], and in the intercalated cells of alkali-loaded rats, AQP6 mRNA and protein levels increased after a week [138]. These results, along with the $\mathrm{pH}$-activation of AQP6 water and anion permeability suggest involvement of AQP6 in acid-base balance in the renal collecting duct. However the lack of in vivo experiments with AQP6 knockouts or naturally occurring mutations precludes any meaningful conclusions about the physiological roles of AQP6.

\section{Ion permeability of AQPs: An unresolved controversy}

AQPs support bulk movements of fluid by giving high water permeability to membranes that secrete osmolytes. These osmolytes are often ions (e.g. $\mathrm{Na}^{+}, \mathrm{K}^{+}$and $\mathrm{Cl}^{-}$) and this is reflected in the fact that several AQPs form macromolecular complexes with ion channels and transporters [41]. Perhaps a more efficient way for nature to achieve this dual permeability would be to have both ions and water pass through the same channel. Indeed it has been observed that several ion channels can transport water [38], but it has also been suggested that some AQPs may function as ion channels.

The $\mathrm{pH}$-sensitive ion permeability of AQP6 is well established, and it is likely that the monomeric pore is the ion pathway given that a point mutation to a pore-lining residue of AQP6 abolished ion permeability [137] and AQP6-mimicking mutations of a pore-lining amino acid residue of AQP5 conferred anion permeability [139]. There is also some evidence that the plant AQP nodulin-26 from soybean can act as a voltage-gated, anion-biased ion channel [140, 141].

More controversial is the idea that the fifth pore formed at the fourfold axis of the tetramer of AQP1 may function as a cation channel. In Xenopus oocytes, heterologous expression of human AQP1 led to a PKA-activated cation permeability probably mediated by phosphorylation of AQP1 [142] and a cGMP-activated cation permeability via direct binding of cGMP to the C-terminus of AQP1 [143]. PKC activity was shown to increase the cation permeability of AQP1 in Xenopus oocytes via direct phosphorylation of AQP1 at residues T157 and T239 [144]. We recently showed that phosphorylation at these sites by PKC is required for trigger-induced translocation of AQP1 to the plasma membrane [145], so this may represent more AQP1 molecules in the membrane rather than an increase in the cation permeability or open probability of a single channel. Phosphorylation of a tyrosine residue (Y253) 
in the C-terminal tail of AQP1 was recently shown to be required for activation by cGMP [146]. The cGMP-activated cation current was verified for purified AQP1 in planar artificial membranes and the AQP1 water permeability inhibitor pCMBS did not inhibit ion permeation, suggesting that the water and ion pathways through AQP1 are not the same[147]. Furthermore, point mutations to residues lining the tetrameric pore altered conductance properties [146]. The open probability was found to be very small $\left(<10^{-6}\right)$ in the planar bilayer system [147], which raises doubts over the physiological relevance of this cation conductance. This study suggested that this very low probability could reflect a misfolding or protein degradation artifact rather than a "real" function of AQP1. Furthermore, human APQ1 expressed in HEK293 cells did not induce an above-background cation conductance when cells were loaded with either cGMP or analogues thereof [148]. Additionally, after the initial report of AQP1 cation permeability by Yool et al, several laboratories reported being unable to replicate this result in the same experimental system (and in some cases, with exactly the same AQP1 construct) [149]. High variability in oocyte response to forskolin was also reported. It was suggested that the discrepancy could be due the protocol for choosing 'healthy' oocytes on which to perform experiments. A cut-off for oocyte membrane potential is routinely used for this. Yool et al choose oocytes with a potential $<-20 \mathrm{mV}$, whereas others used a $-35 \mathrm{mV}$ cut-off. If AQP1 does indeed act as a cation channel it is plausible that it could cause a slight depolarization, leading to oocytes in which AQP1 is acting as an ion channel to be falsely excluded as unhealthy.

Rat choroid plexus cells, which strongly express AQP1, were found to have a cGMPactivated cation conductance that was abolished by treatment with AQP1 siRNA. Activation of the current by atrial natriuretic peptide (ANP), which signals through guanylate cyclase, inhibited basal-to-apical fluid transport. The contribution of AQP1 to this inhibition was not confirmed directly, although $\mathrm{Cd}^{2+}$, which was shown to inhibit cGMP-induced cation conduction of AQP1, reversed the inhibition of fluid transport [150]. ANP and a cGMP analogue were also shown to upregulate apical-tobasal fluid transport in cultured retinal pigment epithelial cells, but this was reversed by an inhibitor of AQP1 water permeability [151].

It is clear that, under the right set of circumstances, AQP1 can act as a cation channel, with the ion pathway probably residing in the central pore formed by tetrameric assembly. Whether or not this ion conductance has physiological relevance remains an open question. However, coupled water and ion movement are vital for several pathophysiological processes including tumour angiogenesis and cell migration [152] and epilepsy [153], so it remains an intriguing possibility that AQPs are performing a dual function.

\section{Cell Volume Regulation by AQPs}

Many cells have the ability to modulate their physical size. This is achieved by the import or export of osmolytes in order to move water into or out of the cell by osmosis. Regulatory cell volume decrease (RVD) is mediated by potassium chloride and taurine efflux and regulatory cell volume increase (RVI) by sodium influx [154]. AQPs play a role in mediating the osmotic water movement in cell volume regulation [155], but there is some evidence that their role may go beyond acting as a passive water pore. 
The stretch-activated transient receptor potential vanilloid type 4 (TRPV4) channel is a $\mathrm{Ca}^{2+}$-biased non-selective cation channel (NSCC) that is activated by cell swelling[156] and has been implicated in cellular responses to osmotic stimuli[157]. In some cell types TRPV4 has been shown to provide a $\mathrm{Ca}^{2+}$ signal that is correlated with activation of the $\mathrm{K}^{+}$and $\mathrm{Cl}^{-}$channels responsible for the decrease in cellular osmolality associated with RVD $[157,158]$. In human and murine salivary gland cells, TRPV4 has a functional interaction with AQP5; in AQP5 knockout cells, the hypotonicity-induced calcium influx through TRPV4 was attenuated and subsequent RVD was abolished. Hypotonicity also increased cell surface expression of both TRPV4 and AQP5 and increased their co-localisation [159]. This suggests a role for AQP5 in the regulation of TRPV4 surface expression or hypotonicity-induced activity.

In another example, the RVD of sperm of AQP3 -/- mice was inhibited compared to wild-type mice and the mice displayed reduced fertility[160] [161]. Upon entry into the female reproductive tract, sperm normally encounter a decrease in extracellular osmolality, which is thought to be the signal that activates sperm motility[160]. However, this hypotonic stress also causes cell swelling which, if left uncorrected by RVD, leads to impaired fertilisation, likely due to excessive bending of the sperm tail inside the uterus [161]. If AQP3 was simply acting passively as a water channel, RVD would not be abolished in AQP3 -/- sperm but rather the timescale on which the cell reaches osmotic equilibrium would be increased. A possible explanation for reduced fertility and altered RVD in AQP3 -/- mice is therefore that AQP3, either alone or as part of a macromolecular complex which is disrupted by AQP3 knockout, is involved in the signalling pathway that activates RVD in sperm.

In a further example, when exposed to a hypotonic extracellular solution, cultured renal cortical collecting duct (CCD) cells, which do not endogenously express AQP2, swelled in proportion to the change in extracellular osmolality but did not exhibit RVD. However, when transfected with AQP2, these cells showed an RVD of approximately $40 \%$. Shrinkage was mediated by $\mathrm{Ca}^{2+}$ influx through TRPV4, which activated $\mathrm{Ca}^{2+}$-dependent $\mathrm{K}^{+}$and $\mathrm{Cl}^{-}$channels and $\mathrm{Ca}^{2+}$-dependent $\mathrm{Ca}^{2+}$ release from intracellular stores. In renal CCD cells expressing AQP2, hypotonic stress caused translocation of TRPV4 to the plasma membrane. This response did not occur in AQP2-negative cells. When TRPV4 was pre-translocated to the cell surface prior to hypotonic exposure, RVD was recovered in AQP2-null cells, showing that it is not simply the high water permeability of AQP2 that allows RVD. However, there did not appear to be any co-localisation between endogenous TRPV4 and overexpressed AQP2 in this system, either before or after hypotonic shock, indicating a functional rather than physical interaction [162]. These observations suggest that AQP2 forms part of a sensory and signalling pathway that results in TRPV4 translocation, possibly via sensing of extracellular osmolality.

In a nasopharyngeal cancer cell line, swelling-induced chloride currents could be inhibited by siRNA-mediated knockdown of AQP3, extracellular application of $\mathrm{CuCl}_{2}$ (a non-specific AQP3 inhibitor) or injection of AQP3 antibodies via recording pipettes. AQP3 also co-immunoprecipitated with the $\mathrm{ClC} 3$ chloride channel in this system [163]. The effect of the current-inhibiting treatments on AQP3-ClC3 interaction or $\mathrm{ClC} 3$ subcellular localization was not investigated, but this data 
suggests a role for AQP3 in RVD chloride efflux in these cells, possibly mediated via activation or localization of the $\mathrm{ClC} 3$ chloride channel.

It has been suggested that AQPs could act as direct sensors of osmotic gradients by coupling conformational changes of the protein to a pressure gradient within the pore [164]. This argument relies on the idea of the hydrostatic pressure within the pore and it is not clear that the application of classical fluid mechanics is applicable to a system typically consisting of $<10$ water molecules. Nonetheless it is an intriguing idea, and taken together, these examples clearly support the idea of a signalling or sensory role for AQPs in cell volume regulation beyond a passive water conduction mechanism.

\section{Regulation of membrane protein localization by AQPs}

There is some evidence that the expression of one AQP can regulate the expression, localization or membrane trafficking of other AQPs and of other membrane proteins.

AQP3 -/- mice have polyuria and a urinary concentrating defect. In the cortical collecting ducts of knockout mice, apical AQP2 expression was reduced compared to controls and basolateral AQP4 localization was completely absent. In contrast, medullary collecting duct levels of both AQP2 and AQP4 was not different between wild-type and AQP3 -/- mice [105]. This may reflect a requirement of AQP3 for expression or trafficking of AQPs 2 and 4 in certain cell types, although these observations could also be due to regulation of AQPs 2 and 4 in response to the polyuria phenotype.

AQP11 -/- mice develop polycystic kidney disease (PKD). In AQP11 -/- mice, polycystin 1 (PC1), a protein to which loss of function mutations are associated with autosomal dominant PKD, was upregulated twofold. However, the plasma membrane localization of PC1 was almost completely abolished and PC1 was differentially glycosylated in the AQP11 -/- mice compared to AQP11 +/+ [165]. Glycosylation of some membrane proteins is a crucial step in protein folding and passage through the ER quality control system [166]. Other membrane proteins (AQP1 and PC2) were correctly glycosylated, suggesting that this was a PC1 specific effect. This data suggests that AQP11 can regulate the surface expression of PC1, possibly by mediating an interaction with a glycosyltransferase or part of the ER quality control system.

The interactions between the stretch-activated cation channel TRPV4 and both AQP2 and AQP5 has been discussed in section 6. Briefly, expression of these AQPs was required for the hypotonicity-induced translocation of TRPV4 to the plasma membrane associated with RVD [159, 162]. It may be that in these systems, AQPs and TRPV4 are present in the same vesicles, but the trafficking machinery interacts only with the AQP. We have recently described hypotonicity-induced translocation of AQP1 in HEK293 cells [145] and AQP4 in primary rat astrocytes [167], so this may reflect a general regulatory mechanism by which AQPs can mediate the subcellular localization of other membrane proteins.

The histone methyltransferase Dot1a was shown to inhibit the expression of AQP5 in murine renal cells. Knocking out Dot1a led to AQP5 expression in these cells. The total level of AQP2 expression was unchanged in the presence of AQP5, but AQP2 membrane trafficking was impaired. In addition to this, co-transfection of AQP5 and 
AQP2 into IMCD3 cells reduced AQP2 surface expression compared to transfection of AQP2 alone, suggesting that AQP5 can regulate AQP2 localization. Furthermore, AQP2 and AQP5 co-immunoprecipitated with one another, suggesting a direct physical interaction [168]. The stoichiometry of the AQP2/AQP5 complexes was not investigated, so it is not clear whether the interaction was between AQP2 and AQP5 homotetramers, or through the formation of AQP2/AQP5 heterotetramers (which is an established regulatory mechanism employed by some plant AQPs [169]).

\section{AQPs and cell-cell adhesion}

Adhesion of cells to one another is a vital process that paved the way for evolution of multicellular organisms, and allows for the formation of the varied and complex structures that make up the anatomy of modern animals and plants.

There is evidence that some AQPs can perform a cell-cell adhesion function by forming a direct interaction with a membrane component on an adjacent cell (possibly another AQP molecule). This is well established for AQP0 and there is controversial evidence that AQP4 may also be able to perform this function.

\subsection{AQP0}

AQP0 (also called MIP, for lens Membrane Intrinsic Protein) is expressed primarily in lens fiber cells, which are cells of the eye specialized to form a tightly-packed transparent layer that scatters a minimal amount of incident light to support the function of the eye [8]. It is the most highly expressed membrane protein in these cells ( $45 \%$ by molarity of all lens fiber membrane protein in mice [170]). AQP0 tetramers assemble into large arrays in junctional microdomains [171], which dynamically associate and dissociate at the array edges, with only an estimated $1 \%$ existing as free tetramers at a given time, measured by high-speed atomic force microscopy of isolated sheep lens fiber membranes [172].

Knockout studies and a variety of naturally-occurring AQP0 mutations in humans and mice [8] consistently show that loss of AQP0 function in the lens fiber cells causes cataracts, likely via loss of the tight packing of the cells required to minimize light scattering. It has also been suggested, based on analysis of lenses of AQP0 -/- and -/+ mice, that AQP0 is vital for creating the lens refractive index gradient required to minimize spherical aberration, via an unknown mechanism [173].

A qualitative analysis of lens fiber cell-cell adhesion in AQP0 -/- mice showed that transgenic expression of AQP1 in the AQP0 -/- model could not rescue fiber adhesion [4], nor correct lens transparency defects [174], supporting the idea that the role of AQP0 in lens physiology is not related to membrane water permeability. Furthermore, expression of AQP0 in several in vitro model systems consistently show that it is able to mediate cell-cell adhesion [175].

Early biophysical work on AQP0 proteoliposomes showed that AQP0 is able to mediate interactions with membranes containing negatively charged lipids [176]. It was suggested that this is due to the presence of several positively charged (and no negatively charged) residues in the extracellular loops A, C and E of AQP0. In human AQP0 this positively charged surface is made up of two arginine residues (R33 and R113) and three histidine residues (H40, H122 and H201). 
A naturally occurring AQP0 mutation, R33C, showed a reduction in cell-cell adhesion of 18,27 or $34 \%$ (depending on methodology) for the mutant when compared to wildtype AQP0, despite no effect on membrane localization [177]. This suggests that removal of the conserved positive charge in loop A has a deleterious effect on AQP0mediated cell adhesion, supporting the idea of a positively charged face of AQP0 that forms electrostatic interactions with negatively charged molecules on an adjacent membrane. It is also possible that addition of the cysteine sulfhydryl group facilitates a novel post-translational modification that inhibits adhesive interactions.

Crystallographic analyses of AQP0 suggest a different model of AQP0 mediated cellcell adhesion in which AQP0 molecules on adjacent cells interact with one another directly. An electron crystal structure of double-layered 2D AQP0 crystals suggested that two AQP0 molecules on adjacent membranes might be able form an interaction via their extracellular loops, primarily mediated by proline residues [178]. Follow-up work showed that the way in which the AQP0 extracellular domains (ECDs) interact in these crystals is strongly dependent on the headgroup of co-crystallized lipids [179]. It has been suggested, based on comparison of the electron structure with a 3D X-ray structure of full-length AQP0 [180], that a conformational change in loop A induced by proteolytic truncation of the C-terminus (which is observed in vivo [8]) could facilitate direct binding of AQP0 molecules to one another [181]. However biochemical analysis showed that truncation of either the $\mathrm{N}$ - or $\mathrm{C}$-terminus has no effect on the adhesion properties of AQP0 [182].

Experiments with a fusion protein consisting of the sequences of the three extracellular loops of AQP0 fused directly adjacent to one another showed that this protein was able to competitively inhibit AQP0 mediated cell-cell adhesion [183]. Given the relative location of the three loops in the AQP0 fold and the lack of transmembrane anchoring in the fusion protein, it is very unlikely that this protein is structurally similar to the ECD of intact AQP0. The fact that, despite this, it functions as an inhibitor of AQP0 mediated cell-cell adhesion does not support the idea of the adhesion being mediated by direct AQP0-AQP0 binding, which would depend on the structure of the ECD. It does, however, support the idea of mediation via an (structure independent) electrostatic interaction between the positively charged AQP0 ECD and a negatively charged membrane component on an adjacent cell.

\subsection{AQP4}

AQP4 is a water-selective AQP primarily expressed in glial cells (astrocytes and retinal Müller cells) of the central nervous system and the basolateral membranes of kidney collecting duct cells [155]. AQP4 exists in two major isoforms (although there is emerging evidence of further isoforms [184]). These are the long (323 amino acids) M1 isoform and the shorter (301 amino acids) M23 isoform, named for the position of the N-terminal methionine. Similarly to AQP0, the M23 isoform of AQP4 is able to form large supramolecular arrays (termed OAPs for Orthogonal Array of Particles).

Electron crystallography of double-layered 2D AQP4-M23 crystals suggested that AQP4 molecules on adjacent cells could interact via their ECDs in a similar way to that suggested for AQP0, again mediated by interaction between proline residues [185]. In the same study, AQP4-M23 was shown to facilitate cell-cell interactions between adhesion-deficient mouse fibroblasts. This result was corroborated in a 
similar experimental system, and it was shown that the adhesion generated by AQP4M23 was considerably weaker than that by AQP0. Furthermore, AQP4-M1 was shown not to facilitate adhesion [175]. Another study in the same experimental system failed to reproduce these results and furthermore failed to find AQP4mediated adhesion in primary mouse glia or AQP4-transfected $\mathrm{CHO}$ cell membrane vesicles [186].

Transfection of AQP4-M23 into a glioblastoma cell line (D54, which lacks endogenous AQP4 expression) increased adhesion of the cells to collagen, fibronectin, laminin, and vitronectin substrates [187]. Clearly this is not due to any potential cell-cell adhesion properties of AQP4 and the lack of substrate specificity makes this a difficult result to interpret. However, AQP4-M23 was shown to preferentially localize to membrane-substrate adhesion sites [188], so it is possible that AQP4-M23 could facilitate aggregation or localization of adhesion molecules, thereby having an indirect effect on cellular adhesion, both to other cells and to basement membrane components.

\section{Conclusion}

Mammalian AQPs are primarily known as facilitators of physiological processes through bi-directional, passive water transport. We have reviewed evidence demonstrating that this is far from all they do. AQPs facilitate physiological processes by mediating the diffusion of small, neutral solutes. This is best understood for AQPmediated glycerol transport that facilitates glycerol metabolism, triglyceride cycling and skin hydration. AQPs also have a role in cell volume regulation and it is becoming increasingly clear that this role can extend beyond simply acting as a passive water pore and may involve AQP-mediated signal transduction, although the mechanisms that may be involved in this are not well understood. Many cell types express several different members of the AQP family and there is emerging evidence that the different members can interact with one another either physically or functionally. The involvement of AQPs in human physiology and cellular homeostasis goes far beyond their acting as simple water pores with implications for a range of physiological processes and pathophysiological conditions.

\section{Acknowledgements}

This work was supported by the Engineering and Physical Sciences Research Council via a Molecular Organisation and Assembly in Cells Doctoral Training Centre studentship to PK (grant no: EP/F500378/1). RMB acknowledges funding from the Biotechnology and Biological Sciences Research Council (BBSRC; via grants BB/I019960/1, BB/K013319/1 and BB/L502194/1) and the Innovative Medicines Joint Undertaking under Grant Agreement $n^{\circ} 115583$ to the ND4BB ENABLE Consortium.

\section{References}

[1] M.L. Kortenoeven, R.A. Fenton, Renal aquaporins and water balance disorders, Biochimica et biophysica acta, 1840 (2014) 1533-1549.

[2] E.A. Nagelhus, O.P. Ottersen, Physiological roles of aquaporin-4 in brain, Physiological reviews, 93 (2013) 1543-1562. 
[3] J. Lebeck, Metabolic impact of the glycerol channels AQP7 and AQP9 in adipose tissue and liver, Journal of molecular endocrinology, 52 (2014) R165178.

[4] S.S. Kumari, S. Eswaramoorthy, R.T. Mathias, K. Varadaraj, Unique and analogous functions of aquaporin 0 for fiber cell architecture and ocular lens transparency, Biochimica et biophysica acta, 1812 (2011) 1089-1097.

[5] J. Badaut, A.M. Fukuda, A. Jullienne, K.G. Petry, Aquaporin and brain diseases, Biochimica et biophysica acta, 1840 (2014) 1554-1565.

[6] L.S. King, M. Yasui, P. Agre, Aquaporins in health and disease, Molecular medicine today, 6 (2000) 60-65.

[7] D. Bockenhauer, D.G. Bichet, Pathophysiology, diagnosis and management of nephrogenic diabetes insipidus, Nature reviews. Nephrology, (2015).

[8] A.B. Chepelinsky, Structural function of MIP/aquaporin 0 in the eye lens; genetic defects lead to congenital inherited cataracts, Handbook of experimental pharmacology, (2009) 265-297.

[9] A. Madeira, T.F. Moura, G. Soveral, Aquaglyceroporins: implications in adipose biology and obesity, Cellular and molecular life sciences : CMLS, 72 (2015) 759-771.

[10] J. Wang, L. Feng, Z. Zhu, M. Zheng, D. Wang, Z. Chen, H. Sun, Aquaporins as diagnostic and therapeutic targets in cancer: how far we are?, Journal of translational medicine, 13 (2015) 96.

[11] D. Ribatti, G. Ranieri, T. Annese, B. Nico, Aquaporins in cancer, Biochimica et biophysica acta, 1840 (2014) 1550-1553.

[12] F. Abascal, I. Irisarri, R. Zardoya, Diversity and evolution of membrane intrinsic proteins, Biochimica et biophysica acta, 1840 (2014) 1468-1481.

[13] B. Yang, A.S. Verkman, Water and glycerol permeabilities of aquaporins 1-5 and MIP determined quantitatively by expression of epitope-tagged constructs in Xenopus oocytes, The Journal of biological chemistry, 272 (1997) 16140-16146. [14] K.L. Nemeth-Cahalan, K. Kalman, J.E. Hall, Molecular basis of pH and Ca2+ regulation of aquaporin water permeability, J Gen Physiol, 123 (2004) 573-580. [15] A.K. Meinild, D.A. Klaerke, T. Zeuthen, Bidirectional water fluxes and specificity for small hydrophilic molecules in aquaporins $0-5$, The Journal of biological chemistry, 273 (1998) 32446-32451.

[16] R.R. Geyer, R. Musa-Aziz, X. Qin, W.F. Boron, Relative CO2/NH3 selectivities of mammalian aquaporins 0-9, Am J Physiol-Cell Ph, 304 (2013) C985-C994. [17] L.M. Holm, T.P. Jahn, A.L. Moller, J.K. Schjoerring, D. Ferri, D.A. Klaerke, T. Zeuthen, NH3 and NH4+ permeability in aquaporin-expressing Xenopus oocytes, Pflugers Archiv : European journal of physiology, 450 (2005) 415-428.

[18] M. Echevarria, E.E. Windhager, S.S. Tate, G. Frindt, Cloning and expression of AQP3, a water channel from the medullary collecting duct of rat kidney, Proceedings of the National Academy of Sciences of the United States of America, 91 (1994) 10997-11001.

[19] A. Herraiz, F. Chauvigne, J. Cerda, X. Belles, M.D. Piulachs, Identification and functional characterization of an ovarian aquaporin from the cockroach Blattella germanica L. (Dictyoptera, Blattellidae), The Journal of experimental biology, 214 (2011) 3630-3638.

[20] J.S. Jung, R.V. Bhat, G.M. Preston, W.B. Guggino, J.M. Baraban, P. Agre, Molecular characterization of an aquaporin cDNA from brain: candidate 
osmoreceptor and regulator of water balance, Proceedings of the National Academy of Sciences of the United States of America, 91 (1994) 13052-13056. [21] S. Raina, G.M. Preston, W.B. Guggino, P. Agre, Molecular cloning and characterization of an aquaporin cDNA from salivary, lacrimal, and respiratory tissues, The Journal of biological chemistry, 270 (1995) 1908-1912.

[22] M. Yasui, A. Hazama, T.H. Kwon, S. Nielsen, W.B. Guggino, P. Agre, Rapid gating and anion permeability of an intracellular aquaporin, Nature, 402 (1999) 184-187.

[23] H. Nagase, J. Agren, A. Saito, K. Liu, P. Agre, A. Hazama, M. Yasui, Molecular cloning and characterization of mouse aquaporin 6 , Biochemical and biophysical research communications, 352 (2007) 12-16.

[24] A. Hazama, D. Kozono, W.B. Guggino, P. Agre, M. Yasui, Ion permeation of AQP6 water channel protein. Single channel recordings after Hg2+ activation, The Journal of biological chemistry, 277 (2002) 29224-29230.

[25] K. Liu, D. Kozono, Y. Kato, P. Agre, A. Hazama, M. Yasui, Conversion of aquaporin 6 from an anion channel to a water-selective channel by a single amino acid substitution, Proceedings of the National Academy of Sciences of the United States of America, 102 (2005) 2192-2197.

[26] L.M. Holm, D.A. Klaerke, T. Zeuthen, Aquaporin 6 is permeable to glycerol and urea, Pflugers Archiv : European journal of physiology, 448 (2004) 181-186. [27] K. Ishibashi, M. Kuwahara, Y. Gu, Y. Kageyama, A. Tohsaka, F. Suzuki, F. Marumo, S. Sasaki, Cloning and functional expression of a new water channel abundantly expressed in the testis permeable to water, glycerol, and urea, The Journal of biological chemistry, 272 (1997) 20782-20786.

[28] K. Liu, H. Nagase, C.G. Huang, G. Calamita, P. Agre, Purification and functional characterization of aquaporin-8, Biology of the cell / under the auspices of the European Cell Biology Organization, 98 (2006) 153-161.

[29] Y. Koyama, T. Yamamoto, D. Kondo, H. Funaki, E. Yaoita, K. Kawasaki, N. Sato, K. Hatakeyama, I. Kihara, Molecular cloning of a new aquaporin from rat pancreas and liver, The Journal of biological chemistry, 272 (1997) 3032930333.

[30] T. Ma, B. Yang, A.S. Verkman, Cloning of a novel water and urea-permeable aquaporin from mouse expressed strongly in colon, placenta, liver, and heart, Biochemical and biophysical research communications, 240 (1997) 324-328. [31] T.P. Jahn, A.L.B. Moller, T. Zeuthen, L.M. Holm, D.A. Klaerke, B. Mohsin, W. Kuhlbrandt, J.K. Schjoerring, Aquaporin homologues in plants and mammals transport ammonia, FEBS letters, 574 (2004) 31-36.

[32] J.M. Carbrey, D.A. Gorelick-Feldman, D. Kozono, J. Praetorius, S. Nielsen, P. Agre, Aquaglyceroporin AQP9: solute permeation and metabolic control of expression in liver, Proceedings of the National Academy of Sciences of the United States of America, 100 (2003) 2945-2950.

[33] K. Ishibashi, T. Morinaga, M. Kuwahara, S. Sasaki, M. Imai, Cloning and identification of a new member of water channel (AQP10) as an aquaglyceroporin, Biochimica et biophysica acta, 1576 (2002) 335-340. [34] M. Ikeda, A. Andoo, M. Shimono, N. Takamatsu, A. Taki, K. Muta, W. Matsushita, T. Uechi, T. Matsuzaki, N. Kenmochi, K. Takata, S. Sasaki, K. Ito, K. Ishibashi, The NPC Motif of Aquaporin-11, Unlike the NPA Motif of Known Aquaporins, Is Essential for Full Expression of Molecular Function, Journal of Biological Chemistry, 286 (2011) 3342-3350. 
[35] K. Yakata, K. Tani, Y. Fujiyoshi, Water permeability and characterization of aquaporin-11, Journal of Structural Biology, 174 (2011) 315-320.

[36] A. Madeira, S. Fernandez-Veledo, M. Camps, A. Zorzano, T.F. Moura, V. Ceperuelo-Mallafre, J. Vendrell, G. Soveral, Human aquaporin-11 is a water and glycerol channel and localizes in the vicinity of lipid droplets in human adipocytes, Obesity, 22 (2014) 2010-2017.

[37] U. Kosinska Eriksson, G. Fischer, R. Friemann, G. Enkavi, E. Tajkhorshid, R. Neutze, Subangstrom resolution X-ray structure details aquaporin-water interactions, Science, 340 (2013) 1346-1349.

[38] A. Horner, F. Zocher, J. Preiner, N. Ollinger, C. Siligan, S.A. Akimov, P. Pohl, The mobility of single-file water molecules is governed by the number of $\mathrm{H}$ bonds they may form with channel-lining residues, Science advances, 1 (2015) e1400083.

[39] E. Tajkhorshid, P. Nollert, M.O. Jensen, L.J. Miercke, J. O'Connell, R.M. Stroud, K. Schulten, Control of the selectivity of the aquaporin water channel family by global orientational tuning, Science, 296 (2002) 525-530.

[40] D. Fu, A. Libson, L.J. Miercke, C. Weitzman, P. Nollert, J. Krucinski, R.M. Stroud, Structure of a glycerol-conducting channel and the basis for its selectivity, Science, 290 (2000) 481-486.

[41] J. Sjohamn, K. Hedfalk, Unraveling aquaporin interaction partners, Biochimica et biophysica acta, 1840 (2014) 1614-1623.

[42] A. Frick, U.K. Eriksson, F. de Mattia, F. Oberg, K. Hedfalk, R. Neutze, W.J. de Grip, P.M. Deen, S. Tornroth-Horsefield, X-ray structure of human aquaporin 2 and its implications for nephrogenic diabetes insipidus and trafficking, Proceedings of the National Academy of Sciences of the United States of America, 111 (2014) 6305-6310.

[43] J.D. Ho, R. Yeh, A. Sandstrom, I. Chorny, W.E. Harries, R.A. Robbins, L.J. Miercke, R.M. Stroud, Crystal structure of human aquaporin 4 at $1.8 \mathrm{~A}$ and its mechanism of conductance, Proceedings of the National Academy of Sciences of the United States of America, 106 (2009) 7437-7442.

[44] J. Jiang, B.V. Daniels, D. Fu, Crystal structure of AqpZ tetramer reveals two distinct Arg-189 conformations associated with water permeation through the narrowest constriction of the water-conducting channel, The Journal of biological chemistry, 281 (2006) 454-460.

[45] T. Walz, T. Hirai, K. Murata, J.B. Heymann, K. Mitsuoka, Y. Fujiyoshi, B.L. Smith, P. Agre, A. Engel, The three-dimensional structure of aquaporin-1, Nature, 387 (1997) 624-627.

[46] M.J. Borgnia, P. Agre, Reconstitution and functional comparison of purified GlpF and AqpZ, the glycerol and water channels from Escherichia coli, Proceedings of the National Academy of Sciences of the United States of America, 98 (2001) 2888-2893.

[47] N. Roudier, P. Bailly, P. Gane, N. Lucien, R. Gobin, J.P. Cartron, P. Ripoche, Erythroid expression and oligomeric state of the AQP3 protein, The Journal of biological chemistry, 277 (2002) 7664-7669.

[48] T. Itoh, T. Rai, M. Kuwahara, S.B. Ko, S. Uchida, S. Sasaki, K. Ishibashi, Identification of a novel aquaporin, AQP12, expressed in pancreatic acinar cells, Biochemical and biophysical research communications, 330 (2005) 832-838. 
[49] D.A. Gorelick, J. Praetorius, T. Tsunenari, S. Nielsen, P. Agre, Aquaporin-11: a channel protein lacking apparent transport function expressed in brain, BMC biochemistry, 7 (2006) 14.

[50] E. Beitz, B. Wu, L.M. Holm, J.E. Schultz, T. Zeuthen, Point mutations in the aromatic/arginine region in aquaporin 1 allow passage of urea, glycerol, ammonia, and protons, Proceedings of the National Academy of Sciences of the United States of America, 103 (2006) 269-274.

[51] Y. Wang, K. Schulten, E. Tajkhorshid, What makes an aquaporin a glycerol channel? A comparative study of AqpZ and GlpF, Structure, 13 (2005) 11071118.

[52] D.F. Savage, J.D. O'Connell, 3rd, L.J. Miercke, J. Finer-Moore, R.M. Stroud, Structural context shapes the aquaporin selectivity filter, Proceedings of the National Academy of Sciences of the United States of America, 107 (2010) 17164-17169.

[53] K. Kishida, H. Kuriyama, T. Funahashi, I. Shimomura, S. Kihara, N. Ouchi, M. Nishida, H. Nishizawa, M. Matsuda, M. Takahashi, K. Hotta, T. Nakamura, S. Yamashita, Y. Tochino, Y. Matsuzawa, Aquaporin adipose, a putative glycerol channel in adipocytes, The Journal of biological chemistry, 275 (2000) 2089620902.

[54] M.T. Skowronski, J. Lebeck, A. Rojek, J. Praetorius, E.M. Fuchtbauer, J. Frokiaer, S. Nielsen, AQP7 is localized in capillaries of adipose tissue, cardiac and striated muscle: implications in glycerol metabolism, American journal of physiology. Renal physiology, 292 (2007) F956-965.

[55] R.E. Duncan, M. Ahmadian, K. Jaworski, E. Sarkadi-Nagy, H.S. Sul, Regulation of lipolysis in adipocytes, Annual review of nutrition, 27 (2007) 79-101.

[56] B.J. Lipworth, Clinical pharmacology of beta 3-adrenoceptors, British journal of clinical pharmacology, 42 (1996) 291-300.

[57] T. Miyauchi, H. Yamamoto, Y. Abe, G.J. Yoshida, A. Rojek, E. Sohara, S. Uchida, S. Nielsen, M. Yasui, Dynamic subcellular localization of aquaporin-7 in white adipocytes, FEBS letters, 589 (2015) 608-614.

[58] M. Vaughan, The production and release of glycerol by adipose tissue incubated in vitro, The Journal of biological chemistry, 237 (1962) 3354-3358. [59] M. Hara-Chikuma, E. Sohara, T. Rai, M. Ikawa, M. Okabe, S. Sasaki, S. Uchida, A.S. Verkman, Progressive adipocyte hypertrophy in aquaporin-7-deficient mice: adipocyte glycerol permeability as a novel regulator of fat accumulation, The Journal of biological chemistry, 280 (2005) 15493-15496.

[60] N. Maeda, T. Funahashi, T. Hibuse, A. Nagasawa, K. Kishida, H. Kuriyama, T. Nakamura, S. Kihara, I. Shimomura, Y. Matsuzawa, Adaptation to fasting by glycerol transport through aquaporin 7 in adipose tissue, Proceedings of the National Academy of Sciences of the United States of America, 101 (2004) 17801-17806.

[61] H. Kondo, I. Shimomura, K. Kishida, H. Kuriyama, Y. Makino, H. Nishizawa, M. Matsuda, N. Maeda, H. Nagaretani, S. Kihara, Y. Kurachi, T. Nakamura, T.

Funahashi, Y. Matsuzawa, Human aquaporin adipose (AQPap) gene. Genomic structure, promoter analysis and functional mutation, European journal of biochemistry / FEBS, 269 (2002) 1814-1826.

[62] U. Laforenza, M.F. Scaffino, G. Gastaldi, Aquaporin-10 represents an alternative pathway for glycerol efflux from human adipocytes, PloS one, 8 (2013) e54474. 
[63] T. Morinaga, M. Nakakoshi, A. Hirao, M. Imai, K. Ishibashi, Mouse aquaporin 10 gene (AQP10) is a pseudogene, Biochemical and biophysical research communications, 294 (2002) 630-634.

[64] E.C. Lin, Glycerol utilization and its regulation in mammals, Annual review of biochemistry, 46 (1977) 765-795.

[65] W.M. Bortz, P. Paul, A.C. Haff, W.L. Holmes, Glycerol turnover and oxidation in man, The Journal of clinical investigation, 51 (1972) 1537-1546.

[66] C.F. Borchgrevink, R.J. Havel, Transport of Glycerol in Human Blood, Proceedings of the Society for Experimental Biology and Medicine. Society for Experimental Biology and Medicine, 113 (1963) 946-949.

[67] J.L. Nelson, M.E. Harmon, R.A. Robergs, Identifying plasma glycerol concentration associated with urinary glycerol excretion in trained humans, Journal of analytical toxicology, 35 (2011) 617-623.

[68] E. Sohara, T. Rai, J. Miyazaki, A.S. Verkman, S. Sasaki, S. Uchida, Defective water and glycerol transport in the proximal tubules of AQP7 knockout mice, American journal of physiology. Renal physiology, 289 (2005) F1195-1200.

[69] C. Goubau, J. Jaeken, E.N. Levtchenko, C. Thys, M. Di Michele, G.A. Martens, E. Gerlo, R. De Vos, G.M. Buyse, N. Goemans, C. Van Geet, K. Freson, Homozygosity for aquaporin $7 \mathrm{G} 264 \mathrm{~V}$ in three unrelated children with hyperglyceroluria and a mild platelet secretion defect, Genet Med, 15 (2013) 55-63.

[70] W.P. Russ, D.M. Engelman, The GxxxG motif: a framework for transmembrane helix-helix association, Journal of molecular biology, 296 (2000) 911-919.

[71] H. Tsukaguchi, C. Shayakul, U.V. Berger, B. Mackenzie, S. Devidas, W.B. Guggino, A.N. van Hoek, M.A. Hediger, Molecular characterization of a broad selectivity neutral solute channel, The Journal of biological chemistry, 273 (1998) 24737-24743.

[72] M. Elkjaer, Z. Vajda, L.N. Nejsum, T. Kwon, U.B. Jensen, M. AmiryMoghaddam, J. Frokiaer, S. Nielsen, Immunolocalization of AQP9 in liver, epididymis, testis, spleen, and brain, Biochemical and biophysical research communications, 276 (2000) 1118-1128.

[73] S.J. Pilkis, D.K. Granner, Molecular physiology of the regulation of hepatic gluconeogenesis and glycolysis, Annual review of physiology, 54 (1992) 885909.

[74] R.A. Fenton, C.L. Chou, G.S. Stewart, C.P. Smith, M.A. Knepper, Urinary concentrating defect in mice with selective deletion of phloretin-sensitive urea transporters in the renal collecting duct, Proceedings of the National Academy of Sciences of the United States of America, 101 (2004) 7469-7474.

[75] G. Calamita, P. Gena, D. Ferri, A. Rosito, A. Rojek, S. Nielsen, R.A. Marinelli, G. Fruhbeck, M. Svelto, Biophysical assessment of aquaporin-9 as principal facilitative pathway in mouse liver import of glucogenetic glycerol, Biology of the cell / under the auspices of the European Cell Biology Organization, 104 (2012) 342-351.

[76] A.M. Rojek, M.T. Skowronski, E.M. Fuchtbauer, A.C. Fuchtbauer, R.A. Fenton, P. Agre, J. Frokiaer, S. Nielsen, Defective glycerol metabolism in aquaporin 9 (AQP9) knockout mice, Proceedings of the National Academy of Sciences of the United States of America, 104 (2007) 3609-3614.

[77] N. Zhang, M.J. Bevan, CD8(+) T cells: foot soldiers of the immune system, Immunity, 35 (2011) 161-168. 
[78] G. Cui, M.M. Staron, S.M. Gray, P.C. Ho, R.A. Amezquita, J. Wu, S.M. Kaech, IL7-Induced Glycerol Transport and TAG Synthesis Promotes Memory CD8+ T Cell Longevity, Cell, 161 (2015) 750-761.

[79] R. Sougrat, M. Morand, C. Gondran, P. Barre, R. Gobin, F. Bonte, M. Dumas, J.M. Verbavatz, Functional expression of AQP3 in human skin epidermis and reconstructed epidermis, The Journal of investigative dermatology, 118 (2002) 678-685.

[80] T. Ma, M. Hara, R. Sougrat, J.M. Verbavatz, A.S. Verkman, Impaired stratum corneum hydration in mice lacking epidermal water channel aquaporin-3, The Journal of biological chemistry, 277 (2002) 17147-17153.

[81] M. Hara, T. Ma, A.S. Verkman, Selectively reduced glycerol in skin of aquaporin-3-deficient mice may account for impaired skin hydration, elasticity, and barrier recovery, The Journal of biological chemistry, 277 (2002) 4661646621.

[82] C.L. Froebe, F.A. Simion, H. Ohlmeyer, L.D. Rhein, J. Mattai, R.H. Cagan, S.E. Friberg, Prevention of Stratum-Corneum Lipid Phase-Transitions Invitro by Glycerol - an Alternative Mechanism for Skin Moisturization, J Soc Cosmet Chem, 41 (1990) 51-65.

[83] S.E. Friberg, I. Kayali, L. Rhein, Direct Role of Linoleic-Acid in Barrier Function - Effect of Linoleic-Acid on the Crystalline-Structure of Oleic-Acid Oleate Model Stratum-Corneum Lipid, J Disper Sci Technol, 11 (1990) 31-47.

[84] M. Hara, A.S. Verkman, Glycerol replacement corrects defective skin hydration, elasticity, and barrier function in aquaporin-3-deficient mice, Proceedings of the National Academy of Sciences of the United States of America, 100 (2003) 7360-7365.

[85] M.D. Norenberg, K.V. Rama Rao, A.R. Jayakumar, Ammonia neurotoxicity and the mitochondrial permeability transition, Journal of bioenergetics and biomembranes, 36 (2004) 303-307.

[86] J.S. Kim, L. He, J.J. Lemasters, Mitochondrial permeability transition: a common pathway to necrosis and apoptosis, Biochemical and biophysical research communications, 304 (2003) 463-470.

[87] F. Martini, J.L. Nath, Anatomy \& physiology, 2nd ed., Benjamin Cummings, San Francisco, 2010.

[88] K. Ishibashi, M. Kuwahara, Y. Gu, Y. Tanaka, F. Marumo, S. Sasaki, Cloning and functional expression of a new aquaporin (AQP9) abundantly expressed in the peripheral leukocytes permeable to water and urea, but not to glycerol, Biochemical and biophysical research communications, 244 (1998) 268-274. [89] K. Ishibashi, S. Sasaki, K. Fushimi, S. Uchida, M. Kuwahara, H. Saito, T. Furukawa, K. Nakajima, Y. Yamaguchi, T. Gojobori, et al., Molecular cloning and expression of a member of the aquaporin family with permeability to glycerol and urea in addition to water expressed at the basolateral membrane of kidney collecting duct cells, Proceedings of the National Academy of Sciences of the United States of America, 91 (1994) 6269-6273.

[90] M. Echevarria, E.E. Windhager, G. Frindt, Selectivity of the renal collecting duct water channel aquaporin-3, The Journal of biological chemistry, 271 (1996) 25079-25082.

[91] S. Jelen, P. Gena, J. Lebeck, A. Rojek, J. Praetorius, J. Frokiaer, R.A. Fenton, S. Nielsen, G. Calamita, M. Rutzler, Aquaporin-9 and urea transporter-A gene deletions affect urea transmembrane passage in murine hepatocytes, American 
journal of physiology. Gastrointestinal and liver physiology, 303 (2012) G12791287.

[92] I.D. Weiner, W.E. Mitch, J.M. Sands, Urea and Ammonia Metabolism and the Control of Renal Nitrogen Excretion, Clinical journal of the American Society of Nephrology : CJASN, (2014).

[93] S.M. Saparov, K. Liu, P. Agre, P. Pohl, Fast and selective ammonia transport by aquaporin-8, The Journal of biological chemistry, 282 (2007) 5296-5301.

[94] B. Yang, Y. Song, D. Zhao, A.S. Verkman, Phenotype analysis of aquaporin-8 null mice, American journal of physiology. Cell physiology, 288 (2005) C11611170.

[95] G. Calamita, D. Ferri, P. Gena, G.E. Liquori, A. Cavalier, D. Thomas, M. Svelto, The inner mitochondrial membrane has aquaporin-8 water channels and is highly permeable to water, The Journal of biological chemistry, 280 (2005) 17149-17153.

[96] B. Yang, D. Zhao, E. Solenov, A.S. Verkman, Evidence from knockout mice against physiologically significant aquaporin 8-facilitated ammonia transport, American journal of physiology. Cell physiology, 291 (2006) C417-423.

[97] A.M. Marini, G. Matassi, V. Raynal, B. Andre, J.P. Cartron, B. Cherif-Zahar, The human Rhesus-associated RhAG protein and a kidney homologue promote ammonium transport in yeast, Nature genetics, 26 (2000) 341-344.

[98] I.D. Weiner, R.T. Miller, J.W. Verlander, Localization of the ammonium transporters, $\mathrm{Rh} \mathrm{B}$ glycoprotein and $\mathrm{Rh} \mathrm{C}$ glycoprotein, in the mouse liver, Gastroenterology, 124 (2003) 1432-1440.

[99] I.D. Weiner, J.W. Verlander, Role of NH3 and NH4+ transporters in renal acid-base transport, American journal of physiology. Renal physiology, 300 (2011) F11-23.

[100] D. Goossens, M.M. Trinh-Trang-Tan, M. Debbia, P. Ripoche, C. VilelaLamego, F. Louache, W. Vainchenker, Y. Colin, J.P. Cartron, Generation and characterisation of Rhd and Rhag null mice, British journal of haematology, 148 (2010) 161-172.

[101] D. Goossens, V. Bony, P. Gane, Y. Colin, J.P. Cartron, Generation of mice with inactivated $\mathrm{Rh}$ or Rhag genes, Transfusion clinique et biologique : journal de la Societe francaise de transfusion sanguine, 13 (2006) 164-166.

[102] L.R. Soria, J. Marrone, G. Calamita, R.A. Marinelli, Ammonia detoxification via ureagenesis in rat hepatocytes involves mitochondrial aquaporin-8 channels, Hepatology, 57 (2013) 2061-2071.

[103] S.M. Molinas, L. Trumper, R.A. Marinelli, Mitochondrial aquaporin-8 in renal proximal tubule cells: evidence for a role in the response to metabolic acidosis, American journal of physiology. Renal physiology, 303 (2012) F458466.

[104] S.M. Molinas, L.R. Soria, J. Marrone, M. Danielli, L. Trumper, R.A. Marinelli, Acidosis-induced downregulation of hepatocyte mitochondrial aquaporin-8 and ureagenesis from ammonia, Biochemistry and cell biology = Biochimie et biologie cellulaire, 93 (2015) 417-420.

[105] T. Ma, Y. Song, B. Yang, A. Gillespie, E.J. Carlson, C.J. Epstein, A.S. Verkman, Nephrogenic diabetes insipidus in mice lacking aquaporin-3 water channels, Proceedings of the National Academy of Sciences of the United States of America, 97 (2000) 4386-4391. 
[106] M. Esbjornsson, J. Bulow, B. Norman, L. Simonsen, J. Nowak, O. Rooyackers, L. Kaijser, E. Jansson, Adipose tissue extracts plasma ammonia after sprint exercise in women and men, Journal of applied physiology, 101 (2006) 15761580.

[107] V. Endeward, J.P. Cartron, P. Ripoche, G. Gros, RhAG protein of the Rhesus complex is a CO2 channel in the human red cell membrane, Faseb J, 22 (2008) 64-73.

[108] M.E. Blank, H. Ehmke, Aquaporin-1 and HCO3(-)-Cl- transporter-mediated transport of $\mathrm{CO} 2$ across the human erythrocyte membrane, The Journal of physiology, 550 (2003) 419-429.

[109] G.M. Preston, T.P. Carroll, W.B. Guggino, P. Agre, Appearance of water channels in Xenopus oocytes expressing red cell CHIP28 protein, Science, 256 (1992) 385-387.

[110] N.L. Nakhoul, B.A. Davis, M.F. Romero, W.F. Boron, Effect of expressing the water channel aquaporin-1 on the $\mathrm{CO} 2$ permeability of Xenopus oocytes, The American journal of physiology, 274 (1998) C543-548.

[111] G.J. Cooper, W.F. Boron, Effect of PCMBS on CO2 permeability of Xenopus oocytes expressing aquaporin 1 or its C189S mutant, The American journal of physiology, 275 (1998) C1481-1486.

[112] V. Endeward, R. Musa-Aziz, G.J. Cooper, L.M. Chen, M.F. Pelletier, L.V. Virkki, C.T. Supuran, L.S. King, W.F. Boron, G. Gros, Evidence that aquaporin 1 is a major pathway for $\mathrm{CO} 2$ transport across the human erythrocyte membrane, Faseb J, 20 (2006) 1974-1981.

[113] B. Yang, N. Fukuda, A. van Hoek, M.A. Matthay, T. Ma, A.S. Verkman, Carbon dioxide permeability of aquaporin-1 measured in erythrocytes and lung of aquaporin-1 null mice and in reconstituted proteoliposomes, The Journal of biological chemistry, 275 (2000) 2686-2692.

[114] G.V. Prasad, L.A. Coury, F. Finn, M.L. Zeidel, Reconstituted aquaporin 1 water channels transport $\mathrm{CO} 2$ across membranes, The Journal of biological chemistry, 273 (1998) 33123-33126.

[115] Y. Wang, J. Cohen, W.F. Boron, K. Schulten, E. Tajkhorshid, Exploring gas permeability of cellular membranes and membrane channels with molecular dynamics, J Struct Biol, 157 (2007) 534-544.

[116] J.S. Hub, B.L. de Groot, Does CO2 permeate through aquaporin-1?, Biophys J, 91 (2006) 842-848.

[117] Y. Wang, S.A. Shaikh, E. Tajkhorshid, Exploring transmembrane diffusion pathways with molecular dynamics, Physiology, 25 (2010) 142-154.

[118] F. Itel, S. Al-Samir, F. Oberg, M. Chami, M. Kumar, C.T. Supuran, P.M. Deen, W. Meier, K. Hedfalk, G. Gros, V. Endeward, CO2 permeability of cell membranes is regulated by membrane cholesterol and protein gas channels, Faseb J, 26 (2012) 5182-5191.

[119] J.S. Hub, F.K. Winkler, M. Merrick, B.L. de Groot, Potentials of mean force and permeabilities for carbon dioxide, ammonia, and water flux across a Rhesus protein channel and lipid membranes, Journal of the American Chemical Society, 132 (2010) 13251-13263.

[120] M. Echevarria, A.M. Munoz-Cabello, R. Sanchez-Silva, J.J. Toledo-Aral, J. Lopez-Barneo, Development of cytosolic hypoxia and hypoxia-inducible factor stabilization are facilitated by aquaporin-1 expression, The Journal of biological chemistry, 282 (2007) 30207-30215. 
[121] G. Li, V. Santoni, C. Maurel, Plant aquaporins: roles in plant physiology, Biochimica et biophysica acta, 1840 (2014) 1574-1582.

[122] R. Kaldenhoff, L. Kai, N. Uehlein, Aquaporins and membrane diffusion of

CO2 in living organisms, Biochimica et biophysica acta, 1840 (2014) 1592-1595.

[123] L.A. Sena, N.S. Chandel, Physiological roles of mitochondrial reactive oxygen species, Molecular cell, 48 (2012) 158-167.

[124] Y. Sheng, I.A. Abreu, D.E. Cabelli, M.J. Maroney, A.F. Miller, M. Teixeira, J.S. Valentine, Superoxide dismutases and superoxide reductases, Chemical reviews, 114 (2014) 3854-3918.

[125] G.P. Bienert, J.K. Schjoerring, T.P. Jahn, Membrane transport of hydrogen peroxide, Biochimica et biophysica acta, 1758 (2006) 994-1003.

[126] M. Reth, Hydrogen peroxide as second messenger in lymphocyte activation, Nature immunology, 3 (2002) 1129-1134.

[127] A. Almasalmeh, D. Krenc, B. Wu, E. Beitz, Structural determinants of the hydrogen peroxide permeability of aquaporins, The FEBS journal, 281 (2014) 647-656.

[128] E.W. Miller, B.C. Dickinson, C.J. Chang, Aquaporin-3 mediates hydrogen peroxide uptake to regulate downstream intracellular signaling, Proceedings of the National Academy of Sciences of the United States of America, 107 (2010) 15681-15686.

[129] M. Hara-Chikuma, S. Chikuma, Y. Sugiyama, K. Kabashima, A.S. Verkman, S. Inoue, Y. Miyachi, Chemokine-dependent T cell migration requires aquaporin-3mediated hydrogen peroxide uptake, The Journal of experimental medicine, 209 (2012) 1743-1752.

[130] F. Vieceli Dalla Sega, L. Zambonin, D. Fiorentini, B. Rizzo, C. Caliceti, L. Landi, S. Hrelia, C. Prata, Specific aquaporins facilitate Nox-produced hydrogen peroxide transport through plasma membrane in leukaemia cells, Biochimica et biophysica acta, 1843 (2014) 806-814.

[131] M.J. Marchissio, D.E. Frances, C.E. Carnovale, R.A. Marinelli, Mitochondrial aquaporin-8 knockdown in human hepatoma HepG2 cells causes ROS-induced mitochondrial depolarization and loss of viability, Toxicology and applied pharmacology, 264 (2012) 246-254.

[132] E.E. Tchekneva, Z. Khuchua, L.S. Davis, V. Kadkina, S.R. Dunn, S. Bachman, K. Ishibashi, E.M. Rinchik, R.C. Harris, M.M. Dikov, M.D. Breyer, Single amino acid substitution in aquaporin 11 causes renal failure, Journal of the American Society of Nephrology : JASN, 19 (2008) 1955-1964.

[133] E.N. Atochina-Vasserman, A. Biktasova, E. Abramova, D.S. Cheng, V.V. Polosukhin, H. Tanjore, S. Takahashi, H. Sonoda, L. Foye, C. Venkov, S.V. Ryzhov, S. Novitskiy, N. Shlonimskaya, M. Ikeda, T.S. Blackwell, W.E. Lawson, A.J. Gow, R.C. Harris, M.M. Dikov, E.E. Tchekneva, Aquaporin 11 insufficiency modulates kidney susceptibility to oxidative stress, American journal of physiology. Renal physiology, 304 (2013) F1295-1307.

[134] G.P. Bienert, F. Chaumont, Aquaporin-facilitated transmembrane diffusion of hydrogen peroxide, Biochimica et biophysica acta, 1840 (2014) 1596-1604. [135] M. Yasui, T.H. Kwon, M.A. Knepper, S. Nielsen, P. Agre, Aquaporin-6: An intracellular vesicle water channel protein in renal epithelia, Proceedings of the National Academy of Sciences of the United States of America, 96 (1999) 58085813. 
[136] T. Ma, B. Yang, W.L. Kuo, A.S. Verkman, cDNA cloning and gene structure of a novel water channel expressed exclusively in human kidney: evidence for a gene cluster of aquaporins at chromosome locus 12q13, Genomics, 35 (1996) 543-550.

[137] M. Ikeda, E. Beitz, D. Kozono, W.B. Guggino, P. Agre, M. Yasui, Characterization of aquaporin- 6 as a nitrate channel in mammalian cells.

Requirement of pore-lining residue threonine 63, The Journal of biological chemistry, 277 (2002) 39873-39879.

[138] D. Promeneur, T.H. Kwon, M. Yasui, G.H. Kim, J. Frokiaer, M.A. Knepper, P. Agre, S. Nielsen, Regulation of AQP6 mRNA and protein expression in rats in response to altered acid-base or water balance, American journal of physiology. Renal physiology, 279 (2000) F1014-1026.

[139] X. Qin, W.F. Boron, Mutation of a single amino acid converts the human water channel aquaporin 5 into an anion channel, American journal of physiology. Cell physiology, 305 (2013) C663-672.

[140] J.W. Lee, Y. Zhang, C.D. Weaver, N.H. Shomer, C.F. Louis, D.M. Roberts, Phosphorylation of nodulin 26 on serine 262 affects its voltage-sensitive channel activity in planar lipid bilayers, The Journal of biological chemistry, 270 (1995) 27051-27057.

[141] C.D. Weaver, N.H. Shomer, C.F. Louis, D.M. Roberts, Nodulin 26, a nodulespecific symbiosome membrane protein from soybean, is an ion channel, The Journal of biological chemistry, 269 (1994) 17858-17862.

[142] A.J. Yool, W.D. Stamer, J.W. Regan, Forskolin stimulation of water and cation permeability in aquaporin 1 water channels, Science, 273 (1996) 12161218.

[143] T.L. Anthony, H.L. Brooks, D. Boassa, S. Leonov, G.M. Yanochko, J.W. Regan, A.J. Yool, Cloned human aquaporin-1 is a cyclic GMP-gated ion channel, Molecular pharmacology, 57 (2000) 576-588.

[144] W. Zhang, E. Zitron, M. Homme, L. Kihm, C. Morath, D. Scherer, S. Hegge, D. Thomas, C.P. Schmitt, M. Zeier, H. Katus, C. Karle, V. Schwenger, Aquaporin-1 channel function is positively regulated by protein kinase $\mathrm{C}$, The Journal of biological chemistry, 282 (2007) 20933-20940.

[145] M.T. Conner, A.C. Conner, C.E. Bland, L.H. Taylor, J.E. Brown, H.R. Parri, R.M. Bill, Rapid aquaporin translocation regulates cellular water flow: mechanism of hypotonicity-induced subcellular localization of aquaporin 1 water channel, The Journal of biological chemistry, 287 (2012) 11516-11525.

[146] E.M. Campbell, D.N. Birdsell, A.J. Yool, The activity of human aquaporin 1 as a cGMP-gated cation channel is regulated by tyrosine phosphorylation in the carboxyl-terminal domain, Molecular pharmacology, 81 (2012) 97-105. [147] S.M. Saparov, D. Kozono, U. Rothe, P. Agre, P. Pohl, Water and ion permeation of aquaporin-1 in planar lipid bilayers. Major differences in structural determinants and stoichiometry, The Journal of biological chemistry, 276 (2001) 31515-31520.

[148] S.P. Tsunoda, B. Wiesner, D. Lorenz, W. Rosenthal, P. Pohl, Aquaporin-1, nothing but a water channel, The Journal of biological chemistry, 279 (2004) 11364-11367.

[149] P. Agre, M.D. Lee, S. Devidas, W.B. Guggino, Aquaporins and ion conductance, Science, 275 (1997) 1490; author reply 1492. 
[150] D. Boassa, W.D. Stamer, A.J. Yool, Ion channel function of aquaporin-1 natively expressed in choroid plexus, The Journal of neuroscience : the official journal of the Society for Neuroscience, 26 (2006) 7811-7819.

[151] N.W. Baetz, W.D. Stamer, A.J. Yool, Stimulation of aquaporin-mediated fluid transport by cyclic GMP in human retinal pigment epithelium in vitro, Investigative ophthalmology \& visual science, 53 (2012) 2127-2132.

[152] S. Saadoun, M.C. Papadopoulos, M. Hara-Chikuma, A.S. Verkman, Impairment of angiogenesis and cell migration by targeted aquaporin-1 gene disruption, Nature, 434 (2005) 786-792.

[153] D.K. Binder, E.A. Nagelhus, O.P. Ottersen, Aquaporin-4 and epilepsy, Glia, 60 (2012) 1203-1214.

[154] E.K. Hoffmann, I.H. Lambert, S.F. Pedersen, Physiology of cell volume regulation in vertebrates, Physiological reviews, 89 (2009) 193-277.

[155] R.E. Day, P. Kitchen, D.S. Owen, C. Bland, L. Marshall, A.C. Conner, R.M. Bill, M.T. Conner, Human aquaporins: regulators of transcellular water flow, Biochimica et biophysica acta, 1840 (2014) 1492-1506.

[156] B. Nilius, J. Vriens, J. Prenen, G. Droogmans, T. Voets, TRPV4 calcium entry channel: a paradigm for gating diversity, American journal of physiology. Cell physiology, 286 (2004) C195-205.

[157] W. Liedtke, Transient receptor potential vanilloid channels functioning in transduction of osmotic stimuli, The Journal of endocrinology, 191 (2006) 515523.

[158] W. Liedtke, C. Kim, Functionality of the TRPV subfamily of TRP ion channels: add mechano-TRP and osmo-TRP to the lexicon!, Cellular and molecular life sciences : CMLS, 62 (2005) 2985-3001.

[159] X. Liu, B.C. Bandyopadhyay, T. Nakamoto, B. Singh, W. Liedtke, J.E. Melvin, I. Ambudkar, A role for AQP5 in activation of TRPV4 by hypotonicity: concerted involvement of AQP5 and TRPV4 in regulation of cell volume recovery, The Journal of biological chemistry, 281 (2006) 15485-15495.

[160] Q. Chen, E.K. Duan, Aquaporins in sperm osmoadaptation: an emerging role for volume regulation, Acta pharmacologica Sinica, 32 (2011) 721-724. [161] Q. Chen, H. Peng, L. Lei, Y. Zhang, H. Kuang, Y. Cao, Q.X. Shi, T. Ma, E. Duan, Aquaporin3 is a sperm water channel essential for postcopulatory sperm osmoadaptation and migration, Cell research, 21 (2011) 922-933.

[162] L. Galizia, A. Pizzoni, J. Fernandez, V. Rivarola, C. Capurro, P. Ford, Functional interaction between AQP2 and TRPV4 in renal cells, Journal of cellular biochemistry, 113 (2012) 580-589.

[163] H. Zhang, H. Li, E. Liu, Y. Guang, L. Yang, J. Mao, L. Zhu, L. Chen, L. Wang, The AQP-3 water channel and the ClC-3 chloride channel coordinate the hypotonicity-induced swelling volume in nasopharyngeal carcinoma cells, The international journal of biochemistry \& cell biology, 57 (2014) 96-107. [164] A.E. Hill, Y. Shachar-Hill, Are Aquaporins the Missing Transmembrane Osmosensors?, The Journal of membrane biology, 248 (2015) 753-765. [165] Y. Inoue, E. Sohara, K. Kobayashi, M. Chiga, T. Rai, K. Ishibashi, S. Horie, X. $\mathrm{Su}$, J. Zhou, S. Sasaki, S. Uchida, Aberrant glycosylation and localization of polycystin-1 cause polycystic kidney in an AQP11 knockout model, Journal of the American Society of Nephrology : JASN, 25 (2014) 2789-2799. 
[166] S.P. Ferris, V.K. Kodali, R.J. Kaufman, Glycoprotein folding and qualitycontrol mechanisms in protein-folding diseases, Disease models \& mechanisms, 7 (2014) 331-341.

[167] P. Kitchen, R.E. Day, L.H. Taylor, M.M. Salman, R.M. Bill, M.T. Conner, A.C. Conner, Identification and molecular mechanisms of the rapid tonicity-induced relocalization of aquaporin 4, The Journal of biological chemistry, (2015). [168] H. Wu, L. Chen, X. Zhang, Q. Zhou, J.M. Li, S. Berger, Z. Borok, B. Zhou, Z. Xiao, H. Yin, M. Liu, Y. Wang, J. Jin, M.R. Blackburn, Y. Xia, W. Zhang, Aqp5 is a new transcriptional target of Dot1a and a regulator of Aqp2, PloS one, 8 (2013) e53342.

[169] C. Hachez, A. Besserer, A.S. Chevalier, F. Chaumont, Insights into plant plasma membrane aquaporin trafficking, Trends in plant science, 18 (2013) 344352.

[170] S. Bassnett, P.A. Wilmarth, L.L. David, The membrane proteome of the mouse lens fiber cell, Molecular vision, 15 (2009) 2448-2463.

[171] S. Scheuring, N. Buzhynskyy, S. Jaroslawski, R.P. Goncalves, R.K. Hite, T. Walz, Structural models of the supramolecular organization of AQP0 and connexons in junctional microdomains, J Struct Biol, 160 (2007) 385-394. [172] A. Colom, I. Casuso, T. Boudier, S. Scheuring, High-speed atomic force microscopy: cooperative adhesion and dynamic equilibrium of junctional microdomain membrane proteins, Journal of molecular biology, 423 (2012) 249256.

[173] S.S. Kumari, K. Varadaraj, Aquaporin 0 plays a pivotal role in refractive index gradient development in mammalian eye lens to prevent spherical aberration, Biochemical and biophysical research communications, 452 (2014) 986-991.

[174] K. Varadaraj, S.S. Kumari, R.T. Mathias, Transgenic expression of AQP1 in the fiber cells of AQP0 knockout mouse: Effects on lens transparency, Experimental eye research, 91 (2010) 393-404.

[175] S.S. Kumari, K. Varadaraj, Intact AQP0 performs cell-to-cell adhesion, Biochemical and biophysical research communications, 390 (2009) 1034-1039. [176] L.F. Michea, M. de la Fuente, N. Lagos, Lens major intrinsic protein (MIP) promotes adhesion when reconstituted into large unilamellar liposomes, Biochemistry, 33 (1994) 7663-7669.

[177] S.S. Kumari, J. Gandhi, M.H. Mustehsan, S. Eren, K. Varadaraj, Functional characterization of an AQP0 missense mutation, R33C, that causes dominant congenital lens cataract, reveals impaired cell-to-cell adhesion, Experimental eye research, 116 (2013) 371-385.

[178] T. Gonen, P. Sliz, J. Kistler, Y. Cheng, T. Walz, Aquaporin-0 membrane junctions reveal the structure of a closed water pore, Nature, 429 (2004) 193197.

[179] R.K. Hite, P.L. Chiu, J.M. Schuller, T. Walz, Effect of lipid head groups on double-layered two-dimensional crystals formed by aquaporin-0, PloS one, 10 (2015) e0117371.

[180] W.E. Harries, D. Akhavan, L.J. Miercke, S. Khademi, R.M. Stroud, The channel architecture of aquaporin 0 at a 2.2-A resolution, Proceedings of the National Academy of Sciences of the United States of America, 101 (2004) 14045-14050. 
[181] A. Engel, Y. Fujiyoshi, T. Gonen, T. Walz, Junction-forming aquaporins, Current opinion in structural biology, 18 (2008) 229-235.

[182] S. Sindhu Kumari, K. Varadaraj, Intact and N- or C-terminal end truncated AQP0 function as open water channels and cell-to-cell adhesion proteins: end truncation could be a prelude for adjusting the refractive index of the lens to prevent spherical aberration, Biochimica et biophysica acta, 1840 (2014) 28622877.

[183] J. Liu, J. Xu, S. Gu, B.J. Nicholson, J.X. Jiang, Aquaporin 0 enhances gap junction coupling via its cell adhesion function and interaction with connexin 50 , Journal of cell science, 124 (2011) 198-206.

[184] M. De Bellis, F. Pisani, M.G. Mola, D. Basco, F. Catalano, G.P. Nicchia, M. Svelto, A. Frigeri, A novel human aquaporin-4 splice variant exhibits a dominantnegative activity: a new mechanism to regulate water permeability, Molecular biology of the cell, 25 (2014) 470-480.

[185] Y. Hiroaki, K. Tani, A. Kamegawa, N. Gyobu, K. Nishikawa, H. Suzuki, T. Walz, S. Sasaki, K. Mitsuoka, K. Kimura, A. Mizoguchi, Y. Fujiyoshi, Implications of the aquaporin-4 structure on array formation and cell adhesion, Journal of molecular biology, 355 (2006) 628-639.

[186] H. Zhang, A.S. Verkman, Evidence against involvement of aquaporin-4 in cell-cell adhesion, Journal of molecular biology, 382 (2008) 1136-1143.

[187] E. McCoy, H. Sontheimer, Expression and function of water channels (aquaporins) in migrating malignant astrocytes, Glia, 55 (2007) 1034-1043. [188] A.J. Smith, B.J. Jin, J. Ratelade, A.S. Verkman, Aggregation state determines the localization and function of M1- and M23-aquaporin-4 in astrocytes, The Journal of cell biology, 204 (2014) 559-573.

[189] W. Humphrey, A. Dalke, K. Schulten, VMD: visual molecular dynamics, Journal of molecular graphics, 14 (1996) 33-38, 27-38. 

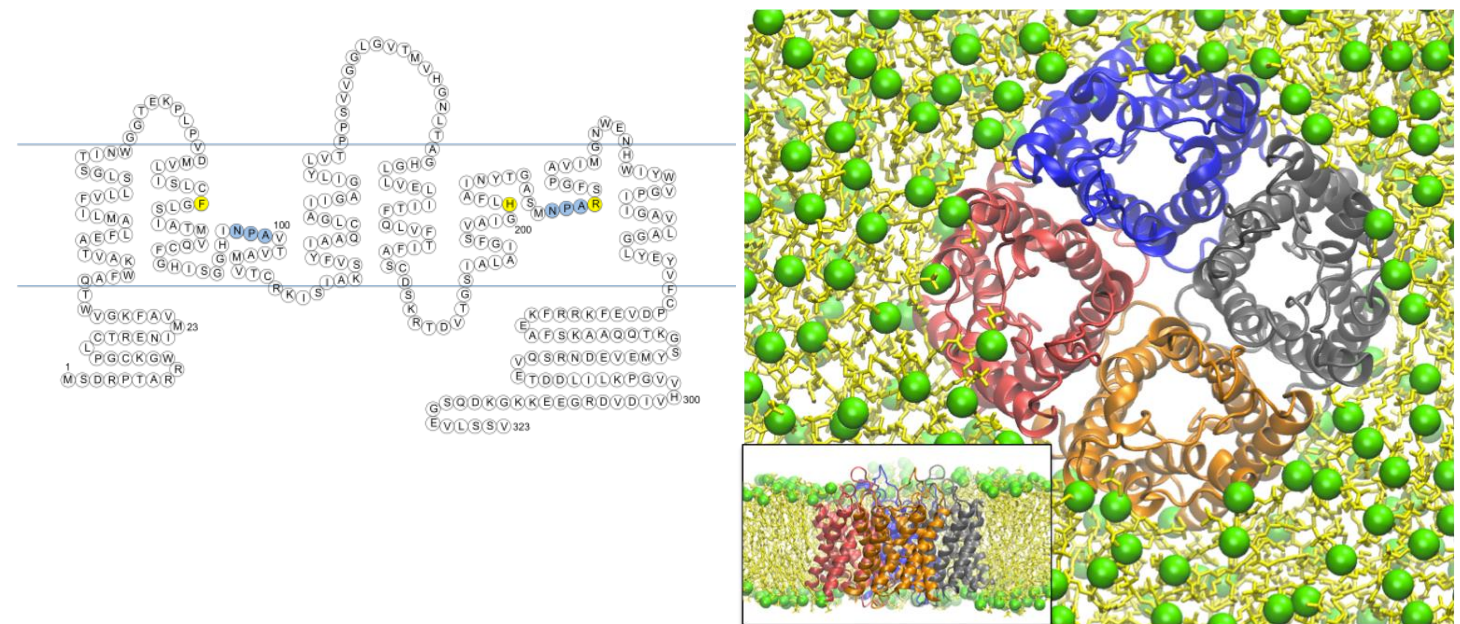

Figure 1 - structure of AQPs exemplified by human AQP4. (A) AQPs have six bilayer-spanning helices and two helix-forming loops. These loops contain the conserved NPA motifs (coloured blue). The residues forming the arginine/aromatic region thought to be important for solute selectivity are coloured yellow. (B) Simulation snapshot of AQP4 embedded in a POPC bilayer. Phosphorus atoms in the lipid headgroups are coloured green and the fatty acid tails are yellow. Visualized using VMD[189]. 